\title{
Test Planning for Modular Testing of Hierarchical SOCs
}

\author{
Krishnendu Chakrabarty, Senior Member, IEEE, Vikram Iyengar, and Mark D. Krasniewski
}

\begin{abstract}
Multilevel test access mechanism (TAM) optimization is necessary for modular testing of hierarchical systems-on-chip (SOCs) that contain older-generation SOCs as embedded megacores. We consider the case where these older-generation SOCs are used as hard cores in new SOC designs, and they are delivered to the system integrator as optimized and technology-mapped layouts. We present three hierarchical test planning and TAM optimization flows that exploit recent advances in TAM design for flattened SOC hierarchies. These techniques are based on the reuse of existing TAM architectures within megacores and the optimization of the top-level TAM under the constraints imposed by "TAM-ed" megacores that are delivered either with or without a wrapper. We present a new megacore wrapper-design technique for the latter case. Unlike prior methods that assume flat test hierarchies, the proposed methods are directly applicable to real-world design-transfer models involving hard megacores between the core vendor and the system integrator for hierarchical SOCs. Experimental results are presented for four ITC'02 SOC test benchmarks that contain megacores.
\end{abstract}

Index Terms-Design transfer and hand-off model, hard cores, megacores, test access mechanism (TAM), TAM optimization, testing time, test wrappers.

\section{INTRODUCTION}

A hierarchical system-on-chip (SOC) is designed by integrating heterogeneous technology cores at several layers of hierarchy [1]. The ability to reuse embedded cores in a hierarchical manner implies that "today's SOC is tomorrow's embedded core" [2]. Two broad design transfer models are emerging in hierarchical SOC design flows.

\section{A. Noninteractive}

The noninteractive design transfer and hand-off model is one in which there is limited communication between the core vendor and the SOC integrator. The hard cores are taken off-the-shelf and integrated into designs as optimized layouts.

Manuscript received August 21, 2003; revised February 29, 2004 and May 7,2004 . This work was supported in part by the National Science Foundation under Grant CCR-9875324 and Grant CCR-0204077, and in part by the Semiconductor Research Corporation under Contract 2004-TJ-1174. A preliminary version of this paper appeared in Proceedings of the IEEE VLSI Test Symposium, pp. 299-304, Napa Valley, CA, April 2003. This paper was recommended by Associate Editor S. M. Reddy.

K. Chakrabarty is with the Department of Electrical and Computer Engineering, Duke University, Durham, NC 27708 USA (e-mail: krish@ee.duke.edu).

V. Iyengar is with IBM Microelectronics, Essex Junction, VT 05452 USA.

M. D. Krasniewski is with the Department of Electrical and Computer Engineering, Purdue University, West Lafayette, IN 47907 USA.

Digital Object Identifier 10.1109/TCAD.2004.842816

\section{B. Interactive}

The interactive design transfer model is typical of larger companies where the business units producing intellectual property (IP) cores may be part of the same organization as the business unit responsible for system integration. Here, there is a certain amount of communication between the core vendor and core user during system integration. The communication of the core user's requirements to the core vendor can play a role in determining the core specifications.

Hierarchical SOCs offer reduced cost and rapid system implementation; however, they pose difficult test challenges. Modular testing of the embedded cores in an SOC can simplify the complex problems of test access and application [3]. For modular testing, an embedded core is isolated from surrounding logic using a test wrapper, and a test access mechanism (TAM) is designed to deliver test data from the input/output (I/O) pins of the SOC. This facilitates the reuse of precomputed tests for individual cores and partitions the SOC for test; thus, the test methodology follows the modular design process.

While several methods for TAM design [4]-[6] and TAM optimization [7]-[9] for modular test have been presented in the literature, the problem of multilevel TAM design and optimization for hierarchical SOCs has not been systematically addressed. In most prior work on TAM design, the SOC hierarchy is assumed to be flattened for the purpose of test [5], [7]-[14]. However, this assumption is often unrealistic in practice, especially when older-generation SOCs are used as hard cores in new SOC designs. In such cases, the core vendor may have already designed a TAM within the "megacore" that is provided as an optimized and technology-mapped layout to the SOC integrator.

A megacore is defined as a design that contains nonmergeable embedded cores. In order to ensure effective testing of an SOC based on megacores, the top-level TAM must communicate with lower-level TAMs within megacores. Moreover, the system-level test architecture must be able to reuse the existing test architecture within cores; redesign of core test structures must be kept to a minimum and it must be consistent with the design transfer model between the core designer and the core user [15].

Three proposals for test access to hierarchical embedded cores were recently presented in [16]-[18]. In [16], the design of a test bus architecture based on scan switches was discussed. However, the TAM hierarchy was limited to a top-level test bus transporting test data to lower-level test buses to which all the cores are connected. Hierarchical TAMs that transport test data to top-level cores and also to lower-level TAMs within megacores were not considered. In [17], the design of a hierarchical TAM was described; however, the lower-level TAMs 
were limited to 1-bit boundary scan chains and multilevel test buses were not considered. In [18], the implementation of a hierarchical test bus architecture was described. However, no attempt was made to optimize these multilevel TAMs to minimize testing time. In particular, none of the three proposals considered the optimization of multilevel TAMs for cores embedded within other cores.

A TAM design methodology that closely follows the design transfer model in use is necessary because if the core vendor has implemented "hard" (i.e., nonalterable) TAMs within megacores, the SOC integrator must take into account these lower-level TAM widths, while optimizing the widths and core assignment for higher-level TAMs. On the other hand, if the core vendor designs TAMs within megacores in consultation with the SOC integrator, the system designer's TAM optimization method must be flexible enough to include parameters for lower-level cores. Finally, multilevel TAM design for SOCs that include reused cores at multiple levels is needed to exploit "TAM reuse" and "wrapper reuse" in the test-development process.

In this paper, we describe the optimization of multilevel TAMs for the "cores within cores" design paradigm. We do not present new algorithms for TAM optimization here; instead, we show how known methods for flattened SOCs can be used for multilevel TAM optimization in hierarchical SOCs. TAM widths are calculated for higher- and lower-level TAMs using a combination of integer linear programming (ILP) and enumeration [7], and efficient heuristics [19]. While other TAM optimizations can also be used in hierarchical design flows, we focus on [7] and [19] because these tools were developed as part of our prior work. Two design flows are presented for the scenario in which megacores are wrapped by the core vendor prior to delivery. In an alternative scenario, we assume that the megacores are delivered to the system integrator in an unwrapped fashion, and the system integrator appropriately designs the megacore wrappers and the SOC-level TAM architecture to minimize the overall testing time.

If the lower-level TAM widths are fixed by the core vendor, the SOC integrator is required to optimize the top-level TAM widths to minimize the testing time under the constraints imposed by the lower-level "TAM-ed and wrapped megacores." In an interactive design transfer model, the SOC integrator can determine a priori an appropriate set of TAM widths across the system hierarchy and communicate lower-level TAM width requirements to the core vendor. The core vendor then appropriately implements the lower-level TAMs and wraps the core before delivery. In another scenario, the core vendor is only required to design and implement a TAM architecture for the megacores; the test wrapper is not designed prior to core delivery. Thus, the core vendor communicates the relevant parameters of the megacore to the SOC integrator, which in turn designs the wrapper for the megacores and optimizes the SOC-level TAM architecture.

Fig. 1 illustrates a megacore that contains four embedded cores and additional logic external to the embedded cores. We assume that the core vendor for this megacore core has wrapped the four embedded cores, and implemented a TAM architecture to access the embedded cores. The TAM architecture consists of two test buses with widths of 3 and 2 bits, respectively, that

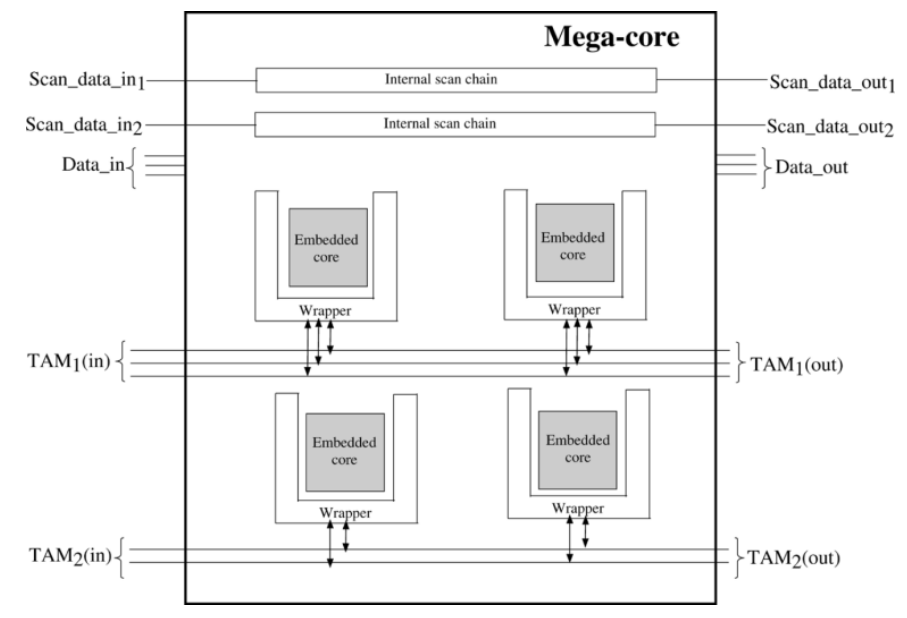

Fig. 1. Illustration of a megacore with a predesigned TAM architecture.

are used to access the four embedded cores. We assume here that the TAM inputs and outputs are not multiplexed with the functional pins. Next, Fig. 2 shows how a two-part wrapper (Wrappers 1 and 2) for the megacore can be designed to drive not only the TAM wires within the megacore, but also to test the logic that is external to the embedded cores. In this design, the TAM inputs for Wrappers 1 and 2 are multiplexed in time, such that the embedded cores within the megacore are tested before the logic external to them, or vice versa. Test generation for the top-level logic is done by the megacore vendor with the wrappers for the embedded cores in functional mode. During the testing of the top-level logic in the megacore using Wrapper 1, the wrappers for the embedded cores must, therefore, be placed in the functional mode to ensure that the top-level logic can be tested completely through the megacore I/Os and scan terminals. While Wrapper 1 can be optimized using existing techniques such as [7] and [20], we formulate a new optimization problem for Wrapper 2 and present a heuristic solution for it in Section III.

The contributions of this paper are twofold. First, it addresses the problem of reusing existing TAMs and wrappers in embedded megacores in hierarchical SOCs. This problem is especially important if megacores with TAMs and wrappers are delivered as hard cores in the form of optimized layouts. In formulating this optimization problem, we not only focus on the reuse of existing test infrastructures, but we also attempt to reuse existing optimization algorithms in a clearly defined optimization flow. For example, consider a megacore (denoted by Core 4) that contains three embedded cores, denoted by Cores $1-3$, respectively. Suppose Cores 1 and 2 are placed on the same (internal) TAM partition within the megacore prior to core delivery. This introduces the constraint that Cores 1 and 2 cannot be tested in parallel. However, a test-planning tool for flat SOCs will evaluate a TAM architecture in which Cores 1 and 2 are placed on different TAM partitions and thereby tested in parallel. If this design yields a lower SOC testing time than a design in which Cores 1 and 2 are placed on the same TAM partition, the tool will incorrectly select the former TAM architecture. Thus, it is important to formulate the problem correctly and provide the right set of constraints to an optimization tool for flat SOCs. 


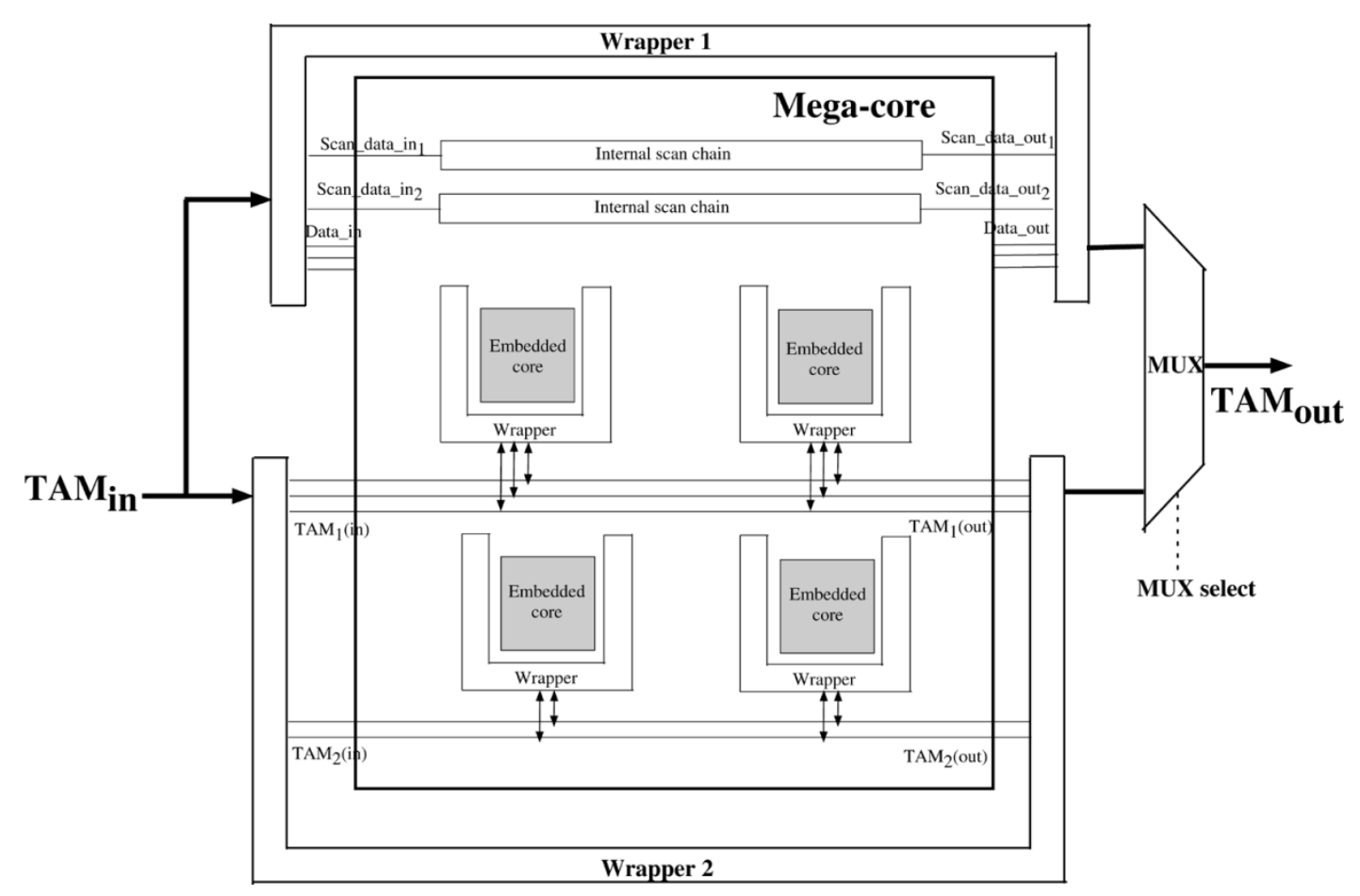

Fig. 2. Illustration of a two-part wrapper for the megacore that is used to drive the TAMs in the megacore and to test the logic external to the embedded cores.

A second contribution of this paper lies in the design of wrappers for embedded megacores during system integration. The bitwidth for test access to be provided to the megacore must be determined on the basis of the test bitwidth needs of the other cores and megacores in the SOC. This problem cannot be solved using a straightforward adaptation of existing wrapper design solutions for flat cores. The distinction between the TAM terminal for the megacores and the scan and data terminals for the top-level logic in the megacore is crucial for wrapper design.

The rest of this paper is organized as follows. In Section II, we formulate the multilevel TAM design problem and show how "flat" TAM-optimization methods can be used to design multilevel TAMs. We also present a new wrapper-design algorithm that can be used by the system integrator when megacores are delivered in unwrapped form. In Section III, we present experimental results for benchmark SOCs [21]. Section IV concludes the paper.

\section{MultileVEl TAM Optimization}

In this section, we present new TAM design flows that are consistent with the design transfer mode between core vendors and SOC integrators. These design flows address the complexity of optimizing TAMs for hierarchical SOCs, while exploiting existing TAM optimization algorithms for "flattened" SOCs. The top-level SOC is composed of embedded cores as well as embedded megacores obtained from core vendors. A megacore may have been a standalone SOC in an earlier generation or is a complex circuit requiring the core vendor to instantiate off-the-shelf cores in its design.

Megacores may be supplied by core vendors in varying degrees of readiness for test integration. For example, the IEEE
P1500 proposal on embedded core test defines two compliance levels for core delivery: 1500-wrapped and 1500-unwrapped [22]. Here we describe three other scenarios, based in part on the P1500 compliance levels. These scenarios refer to the roles played by the system integrator and the core vendor in the design of the TAM and the wrapper for the megacore. For each scenario, the design transfer model refers to the type of information about the megacore that is provided by the core vendor to the system integrator. We use the term wrapped to denote a core for which a wrapper has been predesigned, as in [22]. We use the term TAM-ed to denote a megacore that contains an internal TAM structure.

1) Scenario 1: Not TAM-ed and not wrapped. In this scenario, the system integrator must design a wrapper for the megacore as well as TAMs within the megacore. The megacores are therefore delivered either as soft cores or before final netlist and layout optimization, such that TAMs can be inserted within the megacores. We do not consider this scenario in this paper.

2) Scenario 2: TAM-ed and wrapped. In this scenario, we consider TAM-ed megacores for which wrappers have been designed by the core vendor. This scenario is especially suitable for a megacore that was an SOC in an earlier generation. The width of the TAM that must be supplied to it is prespecified. The I/O and scan chain terminals for wrapped hard-IP subcores may not be available to the SOC integrator to perform further test width adaptation. Such a megacore may also contain its own test control block with strict test protocols specified. Therefore, we assume that such megacores are wrapped by the core vendors prior to design transfer and test data for the megacore cannot be further serialized or parallelized by the SOC integrator. 
This implies that the system integrator has less flexibility in top-level TAM partitioning and core assignment. At the system level, only structures that facilitate normal/test operation, interconnect test, and bypass are created. This scenario includes both the interactive and noninteractive design transfer models.

3) Scenario 3: TAM-ed but not wrapped. In this scenario, the megacore contains lower-level TAMs, but it is not delivered in a wrapped form; therefore, a wrapper for it must be designed by the system integrator. In order to design a wrapper as sketched in Fig. 2, the core vendor must provide information about the number of functional I/Os, the number and lengths of top-level scan chains in the megacore, the number of TAM partitions and the size of each partition, and the testing time for each TAM partition. We assume here that the design transfer model is noninteractive, i.e., the core vendor implements the TAM architecture for the megacores. The extension to the interactive design transfer model for Scenario 3 is straightforward. Compared to the noninteractive design transfer model in Scenario 2, the system integrator in this case has greater flexibility in top-level TAM partitioning and core assignment. Compared to the interactive design transfer model in Scenario 2, the system integrator here has less influence on the TAM design for a megacore; however, this loss of flexibility is somewhat offset by the added freedom of being able to design the megacore wrapper. Width adaptation can be carried out in the wrapper for the megacore such that a narrow TAM at the SOC-level can be used to access a megacore that has a wider internal TAM.

\section{A. Scenario 2: TAM-ed and Wrapped Megacores in the Noninteractive Design Transfer Model}

In the noninteractive design transfer model, the core vendor designs and implements TAM architectures for use within the megacores. Width optimization for these lower-level TAMs is performed without input from the SOC integrator, and testing times for megacores are specified prior to design transfer. The test parameters supplied by the core vendor to the SOC integrator for nonhierarchical cores include the number of primary (including bidirectional) I/Os, test patterns, scan chains, and scan chain lengths. The parameters supplied for the embedded megacores include only the internal TAM width and the testing time.

The multilevel TAM optimization problem in the noninteractive design transfer model for Scenario 2 can now be stated as follows.

$\mathcal{P}_{2-\text { non-int }}$ : Given the test set parameters for the top-level cores and the total TAM width $W$ for the SOC, determine a wrapper design for each core, and a partition of $W$ among the cores in the test schedule, such that the SOC testing time is minimized under the constraints that: 1) $W$ is not exceeded at any time and 2) the megacores receive at least their prespecified TAM widths.

A special case of $\mathcal{P}_{2-\text { non-int }}$ that contains no megacores is equivalent to $\mathcal{P}_{\text {co-opt }}$ in [7]; therefore, $\mathcal{P}_{2-\text { non-int }}$ is $\mathcal{N} \mathcal{P}$-hard.

\section{Design Flow}

Non-interactive_Hierarchical_TAM_Optimizer(SOC)

SOC integrator:

1. For each top-level Core $i$ in the SOC

2. Mark Core $i$ as Started;

3. For each top-level Core $i$ in the SOC

4. If Core $i$ is marked as Started

5. Optimize (Core $i)$;

6. For each top-level Mega-core $i$ in the SOC

7. Obtain testing time $\mathcal{T}_{i}$ and specified TAM width $W_{i}$ :

${ }^{*}$ Let $T_{i}\left(w_{j}\right)$ be the SOC-level testing time of Core $i$ on TAM width $w_{j} * /$

8. Set $T_{i}\left(w_{j}\right)=\mathcal{T}_{i}$, for $w_{j} \geq W_{i}$; Set $T_{i}\left(w_{j}\right)=\infty$, for $w_{j}<W_{i}$;

/* Let $W$ be the total TAM width for the SOC */

9. Partition $W$ among the top-level cores; determine test schedule using $[7,19]$;

10. Implement system-level TAM architecture.

Design Flow Subroutine Optimize(Core $i$ )

Mega-core vendor:

1. Mark Core $i$ as In-progress;

2. For each Core $j$ in Core $i$

3. If Core $j$ is marked as Started

4. Optimize(Core $j$ );

$/^{*}$ Let $W_{i}$ be the total TAM width for Core $i * /$

5. Partition $W_{i}$ among the embedded cores in $i$; determine test schedule using $[7,19]$;

6 . Schedule test for the top-level and interconnect logic within $i$

7. Determine the total testing time $\mathcal{T}_{i}$ for Mega-core $i$;

8. Implement TAM architecture for Core $i$ prior to design transfer.

9. Mark Core $i$ as Done;

Fig. 3. TAM design flow for noninteractive design transfer.

Fig. 3 presents the TAM design flow for hierarchical SOCs in the noninteractive design transfer model. Even though Fig. 3 is not in pseudocode form, it illustrates the overall TAM design flow from core vendor to system integrator. This flow is described using a "Depth-First" [23] hierarchical ordering, such that the TAM design flow goes from the lowest-level cores to the SOC, and the design transfer model is followed at each level in succession.

Lines 1-5 in the design flow describe the start of the DepthFirst ordering of TAM design. Lines 6-10 present the SOC-level TAM design flow (performed by the system integrator). Core test parameters are obtained from the core vendors in the Optimize subroutine. In Lines 8, the TAM width assigned to each 
megacore $i$ is "hardwired" to $W_{i}$ by setting its testing time $T_{i}\left(w_{j}\right)$ to $\mathcal{T}_{i}$ for $w_{j} \geq W_{i}$, and to $\infty$ for $w_{j}<W_{i}$. The result of this TAM width assignment for the TAM design methods [7], [19] used in Line 9 is as follows. The set of $T_{i}\left(w_{j}\right)$ testing time-TAM width variables for each megacore $i$ is reduced to the single constant $\mathcal{T}_{i}$ for $w_{j} \geq W_{i}$, prior to TAM optimization. If $w_{j}<W_{i}, T_{i}\left(w_{j}\right)$ is set to $\infty$. In Line 9 , top-level TAM optimization is carried out for the SOC using a method from [7] and [19] to obtain the final testing time for total TAM width $W$. The system-level TAM architecture is implemented in Line 10.

The Optimize subroutine represents the depth-first TAM design flow for the megacores (performed by the core vendors). The total TAM width $W_{i}$ for each megacore $i$ is determined by the core vendor either from the number of test pins available or from the existing TAM architecture, especially if megacore $i$ was a TAM-ed SOC in a previous generation. In Lines 5-7, the TAM is designed and total testing time $\mathcal{T}_{i}$ for megacore $i$ is determined.

\section{B. Scenario 2: TAM-Ed and Wrapped Megacores in the Interactive Design Transfer Model}

In the interactive design transfer model, the core vendor once again designs and implements TAM architectures for use within the megacores. However, the system integrator is now able to influence the choice of TAM width supplied to megacores by the core vendors based upon system-level TAM width requirements of other cores. The test parameters for each megacore $i$ supplied by the core vendor to the SOC integrator prior to system-level TAM design, therefore, includes a set of 2-tuples $\left\{\left(W_{i}, \mathcal{T}_{i}\right)\right\}$, where each tuple represents a potential TAM width-testing time choice for the megacore, and the number of tuples for each megacore depends on the guidelines from the core user to the core vendor.

The multilevel TAM optimization problem in the interactive design transfer model for Scenario 2 can now be stated as follows.

$\mathcal{P}_{2-\text { int }}$ : Given the test set parameters for the top-level cores and $W$ for the SOC, determine a wrapper design for each core, and a partition of $W$ among the cores in the test schedule, such that the SOC testing time is minimized under the constraints that: 1) $W$ is not exceeded at any time and 2) each megacore receives one of its prespecified TAM widths.

A special case of $\mathcal{P}_{2-i n t}$ in which each megacore has only one prespecified TAM width is equivalent to $\mathcal{P}_{\text {non-int }}$ in Section II-A; therefore, $\mathcal{P}_{2-\text { int }}$ is $\mathcal{N} \mathcal{P}$-hard.

Fig. 4 presents the TAM design flow for the interactive design transfer model. Lines 1-11 represent the SOC-level TAM design flow (performed by the system integrator). In Lines 8 and 9, the set of $T_{i}\left(w_{j}\right)$ variables for each megacore $i$ is reduced to the set of $\mathcal{T}_{i}\left(W_{i}\right)$ values. In Lines 10 and 11, the SOC-level TAM architecture is designed and desired TAM widths for megacores are communicated to core vendors. The core vendors then implement the megacore TAM architectures and transfer their designs to the SOC integrator. The system-level TAM architecture is implemented in Line 13.

The Optimize subroutine contains the depth-first TAM design flow for the megacores (performed by the core vendor). TAM design is now explored for an entire range of width values to

\section{Design Flow}

Interactive_Hierarchical_TAM_Optimizer(SOC)

SOC integrator:

1. For each top-level Core $i$ in the SOC

2. Mark Core $i$ as Started;

3. For each top-level Core $i$ in the SOC

4. If Core $i$ is marked as Started

5. Optimize(Core $i$ );

6. For each top-level Mega-core $i$ in the SOC

7. Obtain the set of values $\left\{\left(W_{i}, \mathcal{T}_{i}\right)\right\}$ from mega-core vendor;

${ }^{*}$ Let $T_{i}\left(w_{j}\right)$ be the SOC-level testing time of Core $i$ on TAM width $w_{j} *$ /

8. For each value of $W_{i}$

9. $\quad$ Set $T_{i}\left(w_{j}\right)=\mathcal{T}_{i}\left(W_{i}\right)$, for $w_{j}=W_{i}$; Set $T_{i}\left(w_{j}\right)=\infty$, for $w_{j} \neq W_{i}$;

${ }^{*}$ Let $W$ be the total TAM width for the $\mathrm{SOC} *$ /

10. Partition $W$ among the cores and determine a test schedule using $[7,19]$;

11. Communicate desired TAM widths for mega-cores to core vendors;

\section{Mega-core vendor:}

12. Implement TAM architectures for mega-cores prior to design transfer;

SOC integrator:

13. Implement system-level TAM architecture.

\section{Design Flow Subroutine Optimize(Core $i)$}

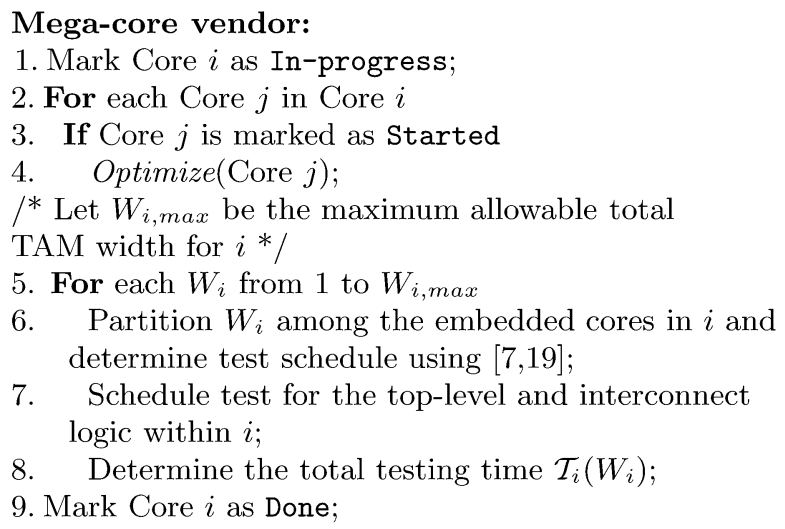

Fig. 4. TAM design flow for interactive design transfer.

estimate potential testing times. While this takes more computation time than a single TAM design calculation, it results in lower SOC testing times as will be seen in Section III. The value of $W_{i, \max }$ is chosen by the core vendor depending on test pin, layout, and overhead constraints for megacore $i$.

\section{Scenario 3: TAM-ed and Unwrapped Megacores}

Recall that in Scenario 3, the megacore vendor implements a TAM architecture for accessing the embedded cores within the megacore. The megacore vendor chooses an appropriate toplevel TAM width, and determines effective TAM partitions and core assignments. (The TAM optimization for the megacores 


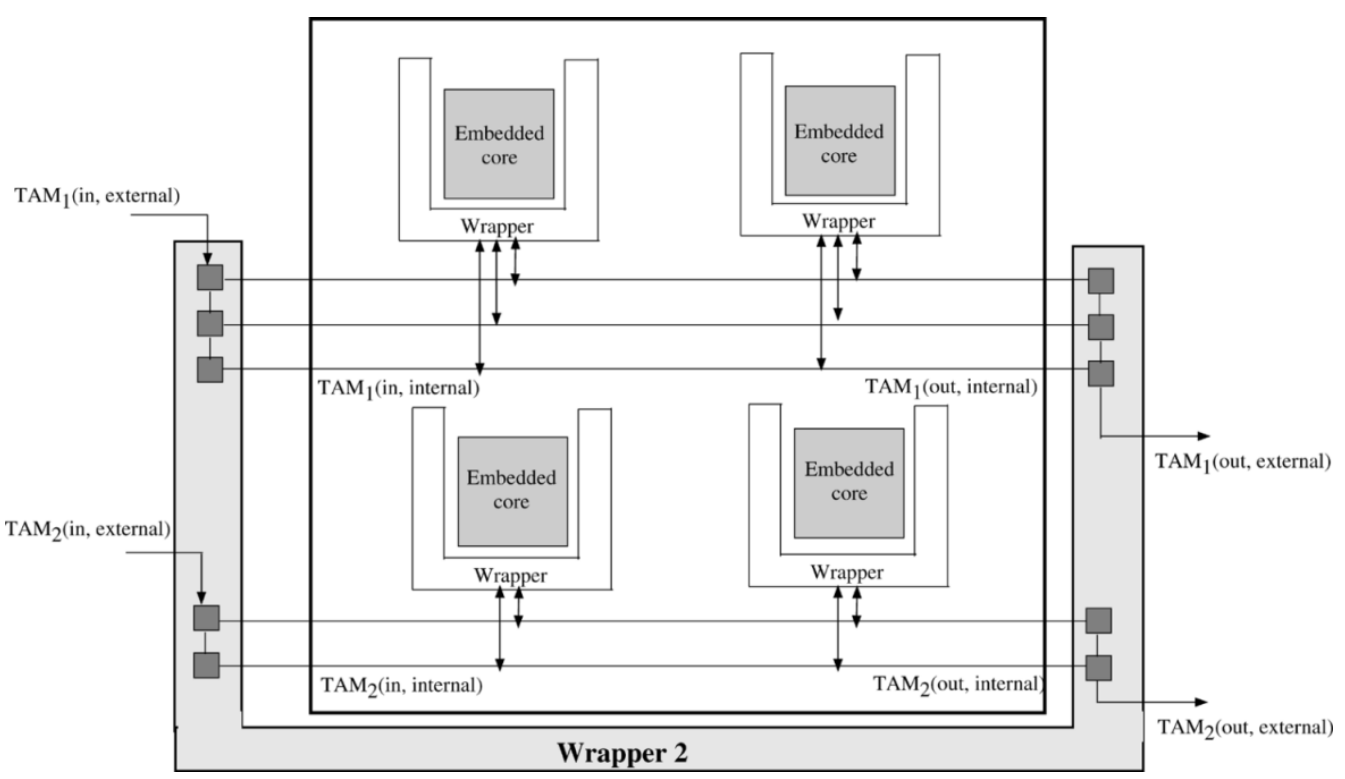

Fig. 5. Illustration of the proposed design of Wrapper 2 for a megacore.

can be carried out by the core vendor using either the interactive or the noninteractive design transfer models.) However, the megacore is delivered unwrapped and the system integrator has the flexibility of designing the wrapper for the megacore. If the top-level TAM width for a TAM-ed megacore is $W_{m}$, the system integrator can provide $W_{m}^{\star}\left(W_{m}^{\star}<W_{m}\right)$ TAM wires to this megacore by designing a wrapper for width adaptation. The testing time for the megacore increases as a result; however, the $W_{m}-W_{m}^{\star}$ TAM wires that are available as a result of width adaptation can now be used to reduce the testing time for other cores on the SOC, potentially leading to lower overall testing time. As indicated earlier, this scenario requires a greater amount of information to be transferred by the megacore vendor to the system integrator.

The multilevel TAM optimization problem under Scenario 3 can now be stated as follows.

$\mathcal{P}_{3}$ : Given the test set parameters for the top-level cores, the necessary parameters for the TAM-ed megacores, and total TAM width $W$ for the SOC, design a wrapper for each core (including each megacore), determine an appropriate number of TAM partitions, divide $W$ among these partitions, and assign the cores on the SOCs to the TAM partitions, such that the SOC testing time is minimized.

A special case of $\mathcal{P}_{3}$ that contains no megacores is equivalent

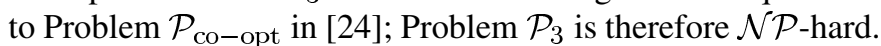

We assume in this paper that the system integrator employs time-domain multiplexing of the TAM partition for the megacore and designs a two-part wrapper as shown in Fig. 2. For a given TAM partition for the megacore, Wrapper 1 can be designed and optimized using known techniques [7], [20]. To optimize Wrapper 2, we use a parallel to serial conversion of the test data stream at the inputs of the megacore's internal TAM, and a similar serial-to-parallel conversion at the outputs of the internal TAM. The basic concept is illustrated in Fig. 5. We refer to this scheme as uniform intra-TAM-partition serialization. While this wrapper design is not provably optimal, the formal development of alternative inter-TAM-partition serialization methods, in which different TAM partitions are chained together, are left for future work. The megacore in Fig. 5 (the logic external to the embedded cores is not shown) has a 5-bit internal TAM, partitioned into two TAMs of widths 3 bits and 2 bits, respectively. For a given limit $W$ on the TAM width at the top-level for the SOC, it might be advantageous to provide only two TAM wires to the megacore, thereby utilizing the remaining wires for the other cores. In such situations, the system integrator can implement Wrapper 2 and the top-level TAM architecture as described next.

Suppose megacore $i$ has a top-level internal TAM width of $W_{i}$ bits and suppose it has $B_{i}$ TAM partitions of widths $\left[w_{i}(1), w_{1}(2), \ldots, w_{i}\left(B_{i}\right)\right]$, respectively. For a given value of SOC-level (external) TAM width $W_{i}^{\star}$ for megacore $i$, where $W_{i}^{\star} \leq W_{i}$, we need to determine an appropriate TAM partition $\left(w_{i}^{\star}(1), w_{i}^{\star}(2), \ldots, w_{i}^{\star}\left(B_{i}\right)\right)$ such that $w_{i}^{\star}(1)+w_{i}^{\star}(2)+\cdots+w_{i}^{\star}\left(B_{i}\right)=W_{i}^{\star}$ and $w_{i}^{\star}(j) \leq w_{i}(j)$, $1 \leq j \leq B_{i}$. Let $T_{i}(j)$ be the total testing time (in clock cycles) for the embedded cores on the internal TAM partition $j$ for megacore $i$. Due to serialization, the testing time for TAM partition $j$ after the megacore is wrapped is $\left\lceil w_{i}(j) / w_{i}^{\star}(j)\right\rceil \cdot T_{i}(j)$ clock cycles. Our goal is to minimize the quantity $\max _{j=1}^{B_{i}}\left\lceil w_{i}(j) / w_{i}^{\star}(j)\right\rceil \cdot T_{i}(j)$. To solve this problem, we enumerate all values of $w_{i}^{\star}(j)$ from 1 to $w_{i}(j)$, while ensuring that $\sum_{j=1}^{B_{i}} w_{i}^{\star}(j)=W_{i}^{\star}$. This approach is computationally feasible because the value of $w_{i}(j)$ is limited by interconnect constraints.

Consider a megacore that has been designed with a top-level TAM of width $W_{i}$, but which is provided with a TAM of width $W_{i}^{\star} \leq W_{i}$ at the SOC level. Fig. 6 describes a procedure to design the wrapper and determine the testing time for this megacore. This procedure is invoked multiple times by the SOC-level TAM design procedure, which considers different values of $W_{i}^{\star}$ during TAM width partitioning.

Fig. 7 presents the TAM design flow for Scenario 3. Lines 1-4 represent the TAM design flow for the megacores as performed by the core vendor. This design is implemented only 


\section{Procedure}

Enumerate_Wrapper_Configurations()

1. $W_{i}$ : Top-level TAM width for Mega-core $i$ designed by mega-core core vendor

2. $W_{i}^{\star}$ : Actual top-level TAM width provided to Mega-core $i$ at the SOC level

3. $B_{i}$ : Number of internal TAM partitions in Mega-core $i$

4. $w_{i}(j)$ : Width of TAM partition $j, 1 \leq j \leq B_{i}$, $\sum_{j=1}^{B_{i}} w_{i}(j)=W_{i}$

5. $T_{i}(j)$ : Testing time of the cores on TAM partition $j$ in Mega-core $i, 1 \leq j \leq B_{i}$

6. For every unique and valid partition of $W_{i}^{\star}$ into $B_{i}$ parts $w_{i}^{\star}(1), w_{i}^{\star}(2), \ldots, w_{i}^{\star}\left(B_{i}\right)$

7. $\quad$ Set $T_{i}^{\star}(j)=\left\lceil\frac{w_{i}(j)}{w_{i}^{\star}(j)}\right\rceil \times T_{i}(j)$

8. Let $T_{i}^{\star}=\max _{j=1}^{B_{i}^{2}} T_{i}(j)$

9. Select $T_{i}^{\star}$ with the smallest value among all partitions of $W_{i}^{\star}$

10. Use Design_wrapper() from [7] to find top-level test time $T_{i}^{\text {top }}$ using external TAM of width $W_{i}^{\star}$ $/ * T_{i}^{W_{i}^{\star}}$ is the testing time for Mega-core $i$ with an external TAM of width $W_{i}^{\star} * /$

11. $T_{i}^{W_{i}^{\star}}=T_{i}^{\star}+T_{i}^{\mathrm{top}}$

Fig. 6. Enumeration of the wrapper configurations in Scenario 3.

for the TAM width determined by the megacore vendor; no test wrapper is designed at this time. $W_{i}$ is determined either from the number of I/O pins available during megacore design or constraints arising from place-and-route issues. Lines 5-10 represent the SOC-level TAM design flow as performed by the system integrator. The system-level TAM architecture is implemented in line 11.

There are a number of additional ways in which multilevel TAM optimization and test scheduling can be carried out for hierarchical SOCs. First, hierarchical TAM optimization can also be carried out using reconfigurable wrappers [25]. The key idea here is to design a wrapper that can be used to interface an embedded core to TAM partitions of different widths. The reconfigurable wrapper requires a small amount of additional hardware and control complexity [25]. For Scenario 2 , a core vendor can design a reconfigurable wrapper that can be exploited to adapt the test bitwidth for a core/megacore during SOC-level test application. In Scenario 3, the system integrator can design a reconfigurable wrapper based on the bitwidth needs of the other cores and megacores in the system. These techniques can be be used to improve upon the work presented in this paper.

Hierarchical TAM optimization and test scheduling can also be carried out by exploiting the presence of multiple instances of embedded cores in an SOC. These cores can be tested using the same test data, and their test bitwidth needs are similar. There-
Design Flow Scenario_3_Hierarchical_TAM_Optimizer()

\section{Mega-core vendor:}

1. For each Mega-core $i$

(starting bottom-up in the hierarchy)

$l^{*}$ Let $W_{i}$ be the total TAM width for $i * /$

2. Partition $W_{i}$ among the embedded cores in $i$ and determine the test schedule using $[7,19]$;

3. Schedule test for the top-level and interconnect logic within $i$

4. Implement TAM architecture for $i$ prior to design transfer;

SOC integrator:

/* Let $W$ be the total TAM width for the SOC */

5 . Obtain the core test parameters for the top-level non-hierarchical and mega-cores;

6. For each Mega-core $i$ do

$/^{*}$ Let $W_{i}^{\star}$ be the number of external TAM lines (to be determined) for Mega-core $i * /$

7. For each possible value of $W_{i}^{\star}, 1 \leq W_{i}^{\star} \leq W_{i}$

8. Apply algorithm Enumerate_Wrapper_Configurations() to Mega-core $i$ and compile the results

$/^{*}$ We have now obtained tuples of the form $\left(\mathcal{T}_{i}^{W_{i}^{\star}}, W_{i}^{\star}\right)^{*} /$

$/^{*}$ Let $B_{i}$ be the number of internal TAMs in Core $i$.

$B_{i}=0$ means that Core $i$ is not a TAM-ed mega-core. */

$/^{*}$ Let $\mathcal{T}_{i}\left(w_{j}\right)$ be the testing time of Mega-core $i$ at the SOC level if it is assigned to a TAM of width $w_{j} * /$

9. $\quad$ Set $\mathcal{T}_{i}\left(w_{j}\right)=\mathcal{T}_{i}^{W_{i}^{\star}}$, for $w_{j} \geq B_{i}$, where $W_{i}^{\star}=w_{j}$

10. Set $\mathcal{T}_{i}\left(w_{j}\right)=\infty$, for $w_{j}<B_{i}$

11. Partition $W$ among the cores using a TAM optimization technique, e.g., from [7,19], and determine the test schedule

12. Implement system-level TAM architecture and mega-core-level test wrappers.

Fig. 7. TAM design flow for Scenario 3.

fore, multiple instances of a core can be connected to the same TAM partition (see Fig. 8). While the test data for these instances can be applied simultaneously via a broadcast scheme as used in [27] for core clustering, the test responses need to be handled in a different manner. For example, space compaction using exclusive-or gates can be used as shown in Fig. 8. Since the wrapper outputs of any instance are not compacted with respect to each other, error masking can be ruled out if no two instances are faulty at the same time. A test-scheduling algorithm at the SOC level can exploit such multiple instances to reduce testing time; however, this approach does not require the reuse of TAMs in embedded megacores. It can, therefore, be combined with the techniques presented in this paper to improve the effectiveness of hierarchical TAM design and test scheduling. In this way, the core-clustering work of [27] can be extended to hierarchical SOCs by taking into account wrapper design for megacores and output-response compaction. 


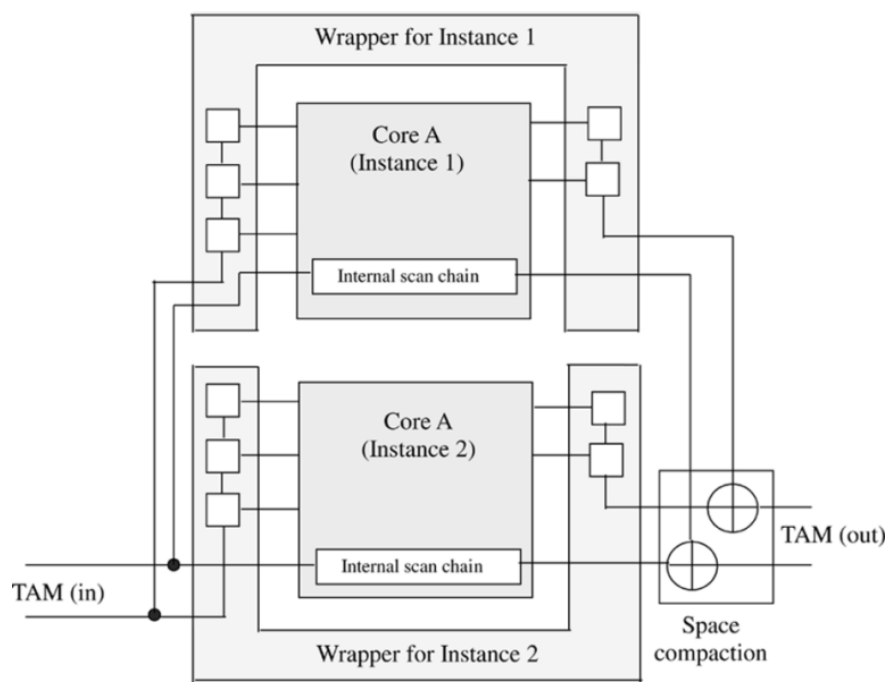

Fig. 8. Exploiting multiple instances of a core for hierarchical TAM optimization and test scheduling.

TABLE I

RESUlTS FOR THE NONINTERACTIVE CASE FOR (a) P22810 USING ILP AND ENUMERATION [7], (b) P34392 USING THE HEURISTIC METHOD OF [19], (c) P93791 Using THE HeURISTIC METHOD, AND (d) A586710 Using ILP AND ENUMERATION

\begin{tabular}{c|c|c|c|c|c|c|c|c|c|c}
\hline & \multicolumn{4}{|c|}{ Method in [7] } & \multicolumn{4}{|c|}{ Hierarchical method } & \multirow{2}{*}{} & \multirow{2}{*}{$W$} \\
\cline { 2 - 9 }$W$ & $\mathcal{T}_{\text {flat }}$ & $\mathcal{E}_{\text {flat }}$ & $B_{\text {flat }}$ & Part $_{\text {flat }}$ & $\mathcal{T}_{\text {hier }}$ & $\mathcal{E}_{\text {hier }}$ & $B_{\text {hier }}$ & Part $_{\text {hier }}$ & $\Delta \mathcal{T}(\%)$ & $\frac{\mathcal{E}_{\text {hier }}}{\mathcal{E}_{\text {flat }}}$ \\
\hline \hline 16 & 462210 & 11 & 2 & 6,10 & 505858 & 31 & 2 & 8,8 & 9.44 & 2.81 \\
24 & 361571 & 24 & 2 & 8,16 & 412682 & 92 & 2 & 8,16 & 14.14 & 3.83 \\
32 & 312659 & 49 & 2 & 10,22 & 396473 & 83 & 2 & 9,23 & 26.81 & 1.69 \\
40 & 278359 & 60 & 2 & 10,30 & 366260 & 77 & 2 & 8,32 & 31.58 & 1.28 \\
48 & 268472 & 84 & 2 & 10,38 & 366260 & 116 & 2 & 8,40 & 36.42 & 1.38 \\
64 & 260638 & 122 & 2 & 10,54 & 366260 & 115 & 2 & 8,56 & 40.52 & 0.94 \\
\hline
\end{tabular}

\begin{tabular}{|c|c|c|c|c|c|c|c|c|c|c|}
\hline \multirow[b]{2}{*}{$W$} & \multicolumn{4}{|c|}{ Method in [19] } & \multicolumn{4}{|c|}{ Hierarchical method } & \multirow[b]{2}{*}{$\Delta \mathcal{T}(\%)$} & \multirow[b]{2}{*}{$\frac{\mathcal{E}_{\text {hie }}}{\mathcal{E}_{\text {fot }}}$} \\
\hline & $\mathcal{T}_{\text {flat }}$ & $\mathcal{E}_{\text {flat }}$ & $B_{\text {flat }}$ & Part $_{\text {flat }}$ & $\mathcal{T}_{\text {hier }}$ & $\mathcal{E}_{\text {hier }}$ & $B_{\text {hier }}$ & Part $_{\text {hier }}$ & & \\
\hline 24 & 729872 & 27 & 4 & $5,6,8$ & 1347023 & 1 & 2 & 23,1 & 84.56 & 0.04 \\
\hline 32 & 663193 & 51 & 5 & $5,4,6,8,9$ & 788873 & 1 & 2 & 16,16 & 18.95 & 0.02 \\
\hline 40 & 544579 & 39 & 4 & $3,7,15,15$ & 728426 & 2 & 3 & $23,16,1$ & 33.76 & 0.05 \\
\hline 48 & 544579 & 205 & 5 & $5,3,8,15,17$ & 618597 & 7 & 3 & $16,16,16$ & 13.59 & 0.03 \\
\hline 56 & 544579 & 109 & 6 & $5,3,5,8,15,20$ & 618597 & 8 & 3 & $24,16,16$ & 13.59 & 0.07 \\
\hline 64 & 544579 & 288 & 6 & $5,3,5,8,15,28$ & 618597 & 9 & 3 & $32,16,16$ & 13.59 & 0.03 \\
\hline
\end{tabular}

\begin{tabular}{|c|c|c|c|c|c|c|c|c|c|c|}
\hline \multirow[b]{2}{*}{$\underline{W}$} & \multicolumn{4}{|c|}{ Method in [19] } & \multicolumn{4}{|c|}{ Hierarchical method } & \multirow[b]{2}{*}{$\Delta \mathcal{T}(\%)$} & \multirow[b]{2}{*}{$\frac{\mathcal{E}_{\text {hies }}}{\mathcal{E}_{\text {flat }}}$} \\
\hline & $\mathcal{T}_{\text {flat }}$ & $\mathcal{E}_{\text {flat }}$ & $B_{\text {flat }}$ & Part flat $_{\text {fl }}$ & $\mathcal{T}_{\text {hier }}$ & $\mathcal{E}_{\text {hier }}$ & $B_{\text {hier }}$ & Part $_{\text {hier }}$ & & \\
\hline 24 & 1209420 & 3 & 3 & $8,8,8$ & 1650880 & 6 & 2 & 4,20 & 36.50 & 2.00 \\
\hline 32 & 894342 & 1 & 2 & 9,23 & 1021320 & 7 & 2 & 16,16 & 14.20 & 7.00 \\
\hline 40 & 741965 & 1 & 3 & $6,10,24$ & 916852 & 7 & 4 & $20,16,2,2$ & 23.57 & 7.00 \\
\hline 48 & 599373 & 3 & 3 & $9,16,23$ & 681816 & 7 & 3 & $16,16,16$ & 13.75 & 2.33 \\
\hline 56 & 514688 & 3 & 3 & $10,23,23$ & 632125 & 7 & 5 & $3,3,16,16,18$ & 22.82 & 2.33 \\
\hline 64 & 473997 & 2 & 3 & $15,23,26$ & 521064 & 8 & 4 & $16,16,16,16$ & 9.93 & 4.00 \\
\hline
\end{tabular}

\begin{tabular}{c|c|c|c|c|c|c|c|c|c|c}
\hline & \multicolumn{3}{|c|}{ Method in [7] } & \multicolumn{3}{c|}{ Hierarchical method } & \multirow{2}{*}{} \\
\cline { 2 - 9 }$W$ & $\mathcal{T}_{\text {flat }}$ & $\mathcal{E}_{\text {flat }}$ & $B_{\text {flat }}$ & Part $_{\text {flat }}$ & $\mathcal{T}_{\text {hier }}$ & $\mathcal{E}_{\text {hier }}$ & $B_{\text {hier }}$ & Part $_{\text {hier }}$ & $\Delta \mathcal{T}(\%)$ & $\frac{\mathcal{E}_{\text {hiar }}}{\mathcal{E}_{\text {flat }}}$ \\
\hline \hline 16 & $4.15 \times 10^{7}$ & 9 & 2 & 8,8 & $4.44 \times 10^{7}$ & 21 & 2 & 2,14 & 6.99 & 2.33 \\
24 & $2.84 \times 10^{7}$ & 7 & 2 & 8,16 & $3.06 \times 10^{7}$ & 14 & 2 & 8,16 & 7.75 & 2.00 \\
32 & $2.10 \times 10^{7}$ & 2 & 2 & 8,24 & $2.88 \times 10^{7}$ & 19 & 2 & 8,24 & 37.14 & 9.50 \\
40 & $1.91 \times 10^{7}$ & 4 & 2 & 10,30 & $2.50 \times 10^{7}$ & 26 & 2 & 8,32 & 30.89 & 4.21 \\
48 & $1.52 \times 10^{7}$ & 4 & 2 & 10,38 & $2.14 \times 10^{7}$ & 41 & 2 & 8,40 & 40.79 & 10.25 \\
56 & $1.41 \times 10^{7}$ & 4 & 2 & 10,46 & $2.14 \times 10^{7}$ & 42 & 2 & 8,48 & 51.77 & 10.50 \\
64 & $1.41 \times 10^{7}$ & 5 & 2 & 10,54 & $2.14 \times 10^{7}$ & 115 & 2 & 8,56 & 51.77 & 23.00 \\
\hline
\end{tabular}

The use of exclusive-or gates for output compaction does not provide diagnostic information to identify the failing instance. To obtain more fine-grained diagnostic information, a follow-up test session can be used to target only the "candidate" failing cores.
An alternative technique to handle identical core instances is presented in [26]. The key idea here is to use a broadcast mode for pattern application and use bit-wise comparison on the outputs. The bit-wise comparison is carried out using exclusive-or gates and "1-detect" cells on the scan outputs. A drawback of this approach is that it requires modifications to the P1500 wrapper, which is expected to become an IEEE standard soon. A single wrapper with the exclusive-or gates and 1-detect logic is required in [26] for all core instances. Therefore, this method is not feasible if the core instances are delivered in a wrapped form to the system integrator. Diagnosis of failing core instances requires that the scan chains of the cores be multiplexed out to chip pins.

\section{EXPERIMENTAL RESULTS}

In this section, we present experimental results for two multilevel TAM design scenarios (Scenarios 2 and 3). For Scenario 2, we consider both interactive and noninteractive design transfer models. The experimental results are presented for four SOCs: p22810, p34392, p93791, and a586710 from the ITC'02 SOC test benchmarks [21]. These SOCs are appropriate for these experiments because they are hierarchical, containing multiple levels of embedded cores. The experimental results were obtained using a Sun Ultra 10 workstation with a $333-\mathrm{MHz}$ processor and $256 \mathrm{MB}$ of memory.

\section{A. Noninteractive Design Transfer Model}

We first performed TAM optimization using the design flow in Fig. 3. In Table I, we compare the testing times (in clock cycles) and CPU times (in seconds) of the proposed hierarchical TAM optimization method with those of the corresponding "flat" methods in [7] and [19]. Although hierarchical TAM optimization was performed based on both methods in [7] and [19] for each SOC, representative results are presented here for only one of the two methods for each SOC. (Similar results are obtained for the cases not shown here.) The testing times for the "flat" and hierarchical methods are denoted by $\mathcal{T}_{\text {flat }}$ and $\mathcal{T}_{\text {hier }}$, respectively. The number of SOC-level TAM partitions for the "flat" and hierarchical methods are denoted by $B_{\text {flat }}$ and $B_{\text {hier }}$, respectively. Similarly, the CPU times for the "flat" and hierarchical methods are denoted by $\mathcal{E}_{\text {flat }}$ and $\mathcal{E}_{\text {hier }}$, respectively. The percentage change in testing time $\Delta \mathcal{T}$ using hierarchical TAM optimization is calculated as $\left(\left(\mathcal{T}_{\text {hier }}-\mathcal{T}_{\text {flat }}\right) / \mathcal{T}_{\text {flat }}\right) \times 100$.

In Table I(a), we present results for $\mathrm{p} 22810$. The hierarchical TAM optimization flow was based on the ILP and enumeration method of [7]. TAM widths supplied to each megacore were fixed at eight bits prior to system-level TAM design $\left(W_{i}=\right.$ $W_{i}^{\star}=8$ for megacore $i$ ). While the testing times obtained are higher than those obtained by (unrealistically) assuming that the SOC hierarchy can be flattened, the results obtained here are more realistic for hierarchical TAM design. Note from Table I(a) that the testing time for the hierarchical method levels out at 366260 cycles at $W=40$. A total top-level TAM width of 40 is, therefore, an effective choice for this SOC.

In Table I(b), we present results for p34392. Hierarchical TAM optimization was performed based on the heuristic method of [19]. A TAM width of 16 bits $\left(W_{i}=W_{i}^{\star}=16\right.$ for megacore 
TABLE II

RESULTS FOR THE INTERACTIVE CASE FOR (a) P22810 USING ILP AND ENUMERATION [7], (b) P34392 USING THE HEURISTIC METHOD OF [19], (c) P93791 Using THE HeURISTIC METHOD, AND (d) A586710 USING ILP AND ENUMERATION

\begin{tabular}{c|c|c|c|c|c|c|c}
\hline & \multicolumn{4}{|c|}{ Hierarchical method } & \multicolumn{2}{c|}{ Compared to [7] } & Compared to non- \\
$W$ & $\mathcal{T}_{\text {hier }}$ & $\mathcal{E}_{\text {hier }}$ & $B_{\text {hier }}$ & Part $_{\text {hier }}$ & $\Delta \mathcal{T}(\%)$ & $\frac{\text { hier }}{\mathcal{E}_{\text {flat }}}$ & interactive: $\Delta \mathcal{T}(\%)$ \\
\hline \hline 16 & 483795 & 23 & 2 & 5,11 & 4.66 & 2.09 & -4.36 \\
24 & 363812 & 56 & 2 & 9,15 & 0.62 & 2.33 & -11.84 \\
32 & 324079 & 120 & 2 & 14,18 & 3.65 & 2.45 & -18.26 \\
40 & 288159 & 79 & 2 & 9,31 & 3.52 & 1.32 & -21.32 \\
48 & 272892 & 129 & 2 & 17,31 & 1.65 & 1.54 & -25.49 \\
64 & 263443 & 187 & 2 & 29,35 & 1.08 & 1.53 & -28.07 \\
\hline
\end{tabular}

\begin{tabular}{|c|c|c|c|c|c|c|c|}
\hline \multirow[b]{3}{*}{$W$} & & & & (a) & & & \\
\hline & \multicolumn{4}{|c|}{ Hierarchical method } & \multicolumn{2}{|c|}{ Compared to [19] } & \multirow{2}{*}{$\begin{array}{l}\text { Compared to non- } \\
\text { interactive: } \Delta \mathcal{T}(\%)\end{array}$} \\
\hline & $\mathcal{T}_{\text {hier }}$ & $\mathcal{E}_{\text {hier }}$ & $B_{\text {hier }}$ & Part $_{\text {hier }}$ & $\Delta \mathcal{T}(\%)$ & $\frac{\mathcal{E}_{\text {hier }}}{\mathcal{E}_{\text {flat }}}$ & \\
\hline 24 & 819176 & 1 & 3 & $10,9,5$ & 12.24 & 0.04 & -39.18 \\
\hline 32 & 713174 & 1 & 3 & $22,5,5$ & 7.54 & 0.02 & -9.60 \\
\hline 40 & 606261 & 2 & 3 & $17,17,6$ & 11.33 & 0.05 & -16.77 \\
\hline 48 & 593925 & 4 & 3 & $25,17,6$ & 9.06 & 0.02 & -3.98 \\
\hline 56 & 581588 & 4 & 3 & $30,20,6$ & 6.79 & 0.04 & -5.98 \\
\hline 64 & 581588 & 4 & 3 & $38,20,6$ & 6.79 & 0.01 & -5.98 \\
\hline
\end{tabular}

\begin{tabular}{c|c|c|c|c|c|c|c}
\hline & \multicolumn{4}{|c|}{ Hierarchical method } & \multicolumn{2}{c|}{ Compared to [19] } & \multirow{2}{*}{$\begin{array}{c}\text { Compared to non- } \\
W\end{array}$} \\
\cline { 2 - 6 } & $\mathcal{T}_{\text {hier }}$ & $\mathcal{E}_{\text {hier }}$ & $B_{\text {hier }}$ & Part $_{\text {hier }}$ & $\Delta \mathcal{T}(\%)$ & $\frac{\mathcal{E}_{\text {lier }}}{\mathcal{E}_{\text {flat }}}$ & interactive: $\Delta \mathcal{T}(\%)$ \\
\hline \hline 16 & 1927010 & 8 & 5 & $4,3,3,3,3$ & 7.88 & 4.00 & 5.73 \\
24 & 1293990 & 14 & 4 & $3,4,8,9$ & 6.99 & 4.67 & -4.27 \\
32 & 863765 & 22 & 3 & $24,5,3$ & -3.42 & 22.00 & -20.56 \\
40 & 758156 & 37 & 2 & 24,16 & 2.18 & 37.00 & -9.72 \\
48 & 635710 & 68 & 2 & 24,24 & 6.06 & 22.67 & -24.30 \\
64 & 481896 & 174 & 2 & 48,16 & 1.67 & 87 & -42.62 \\
\hline
\end{tabular}

\begin{tabular}{c|c|c|c|c|c|c|c}
\hline \multirow{7}{*}{$W$} & \multicolumn{9}{|c|}{ Hierarchical method } & \multicolumn{2}{c|}{ Compared to [7] } & \multirow{2}{*}{$\begin{array}{c}\text { Compared to non- } \\
\text { interactive: } \Delta \mathcal{T}(\%)\end{array}$} \\
\cline { 2 - 7 } & $\mathcal{T}$ & $\mathcal{E}$ & $B$ & Part & $\Delta \mathcal{T}(\%)$ & $\mathcal{E}_{\text {hier }}$ & (c) \\
\hline \hline 24 & $3.06 \times 10^{7}$ & 30 & 2 & 16,8 & 7.75 & 4.29 & 0.00 \\
32 & $2.11 \times 10^{7}$ & 24 & 3 & $18,9,5$ & 0.48 & 12.00 & -26.74 \\
40 & $1.91 \times 10^{7}$ & 40 & 3 & $21,12,7$ & 0.00 & 10.00 & -23.60 \\
48 & $1.75 \times 10^{7}$ & 45 & 3 & $23,16,9$ & 15.13 & 11.25 & -18.22 \\
56 & $1.70 \times 10^{7}$ & 46 & 3 & $26,21,9$ & 20.57 & 11.50 & -20.57 \\
64 & $1.63 \times 10^{7}$ & 56 & 3 & $37,18,9$ & 15.60 & 11.2 & -23.83 \\
\hline
\end{tabular}

i) was supplied to each megacore prior to system-level TAM optimization. The increase in testing time over the "flat" case is between $13.59 \%$ and $84.56 \%$. The testing time for the flat method reaches a minimum value of 544579 cycles at $W=40$. This lower bound (observed earlier in [7], [9], and [19]) is the minimum testing time for Core 18 (the bottleneck core). For the hierarchical method, the testing time does not decrease below 618597 cycles. This is because Module 18, which is a megacore in p34292, has a testing time of 618597 clock cycles with an internal TAM width of 16 bits. We therefore achieve the lower bound for the SOC testing time using hierarchical TAM optimization. The CPU times for the hierarchical method are significantly lower than those for the flat method. This is because in the flat method, an ILP model is run as a final step for all 19 cores of p34392 simultaneously, thereby taking longer CPU time. In the hierarchical optimization flow, the SOC is partitioned per megacore and the ILP model runs significantly faster.
TABLE III

RESULTS FOR SCENARIO 3 FOR (a) P22810 USING ILP AND ENUMERATION [7], (b) P34392 USING THE HEURISTIC METHOD OF [19], (c) P93791 USING THE HEURISTIC METHOD, AND (d) A586710 USING ILP AND ENUMERATION

\begin{tabular}{|c|c|c|c|c|c|c|c|c|}
\hline \multirow[b]{2}{*}{$W$} & \multicolumn{2}{|c|}{ Method in [7] } & \multicolumn{4}{|c|}{ Hierarchical method } & \multirow[b]{2}{*}{$\Delta \mathcal{T}(\%)$} & \multirow[b]{2}{*}{$\frac{\mathcal{E}_{\text {hier }}}{\mathcal{E}_{\text {flat }}}$} \\
\hline & $\mathcal{T}_{\text {flat }}$ & $\mathcal{E}_{\text {flat }}$ & $\mathcal{T}_{\text {hier }}$ & $\mathcal{E}_{\text {hier }}$ & $B_{\text {hier }}$ & Part $_{\text {hier }}$ & & \\
\hline 16 & 462210 & 11 & 497079 & 59 & 2 & 7,9 & 7.54 & 5.36 \\
\hline 24 & 361571 & 24 & 408317 & 97 & 2 & 8,16 & 12.93 & 4.04 \\
\hline 32 & 312659 & 49 & 392106 & 101 & 2 & 9,23 & 25.41 & 2.06 \\
\hline 40 & 278359 & 60 & 361895 & 136 & 2 & 8,32 & 30.01 & 2.27 \\
\hline 48 & 268472 & 84 & 361894 & 171 & 2 & 10,38 & 35.80 & 2.04 \\
\hline 56 & 266800 & 80 & 361894 & 169 & 2 & 10,46 & 35.64 & 2.11 \\
\hline 64 & 260638 & 122 & 361894 & 199 & 2 & 10,54 & 38.85 & 1.63 \\
\hline
\end{tabular}

\begin{tabular}{c|c|c||c|c|c|c|c|c}
\hline & \multicolumn{2}{|c|}{ Method in $[19]$} & \multicolumn{5}{|c|}{ Hierarchical method } & \multirow{2}{*}{} \\
\cline { 2 - 6 }$W$ & $\mathcal{T}_{\text {flat }}$ & $\mathcal{E}_{\text {flat }}$ & $\mathcal{T}_{\text {hier }}$ & $\mathcal{E}_{\text {hier }}$ & $B_{\text {hier }}$ & Part $_{\text {hier }}$ & $\Delta \mathcal{T}(\%)$ & $\frac{\mathcal{E}_{\text {hier }}}{\mathcal{E}_{\text {flat }}}$ \\
\hline \hline 16 & 1033210 & 27 & 1192930 & 3 & 2 & 7,9 & 15.46 & 0.11 \\
24 & 729872 & 27 & 829938 & 3 & 3 & $5,9,10$ & 13.71 & 0.11 \\
32 & 663193 & 51 & 788873 & 3 & 2 & 16,16 & 18.95 & 0.06 \\
40 & 544579 & 39 & 618597 & 3 & 3 & $6,16,18$ & 13.59 & 0.08 \\
48 & 544579 & 205 & 618597 & 4 & 3 & $6,16,26$ & 13.59 & 0.02 \\
56 & 544579 & 109 & 618597 & 6 & 3 & $6,16,34$ & 13.59 & 0.06 \\
64 & 544579 & 288 & 618597 & 8 & 3 & $6,16,42$ & 13.59 & 0.03 \\
\hline
\end{tabular}

\begin{tabular}{|c|c|c|c|c|c|c|c|c|}
\hline \multirow[b]{2}{*}{$W$} & \multicolumn{2}{|c|}{ Method in [19] } & \multicolumn{4}{|c|}{ Hierarchical method } & \multirow[b]{2}{*}{$\Delta \mathcal{T}(\%)$} & \multirow[b]{2}{*}{$\frac{\mathcal{E}_{\text {hier }}}{\mathcal{E}_{\text {flat }}}$} \\
\hline & $\mathcal{T}_{\text {flat }}$ & $\mathcal{E}_{\text {flat }}$ & $\mathcal{T}_{\text {hier }}$ & $\mathcal{E}_{\text {hier }}$ & $B_{\text {hier }}$ & Part $_{\text {hier }}$ & & \\
\hline 16 & 1786200 & 2 & 1829530 & 12 & 4 & $3,3,5,5$ & 2.43 & 6.00 \\
\hline 24 & 1209420 & 3 & 1232060 & 11 & 2 & 8,16 & 1.87 & 3.67 \\
\hline 32 & 894342 & 1 & 915082 & 11 & 3 & $7,9,16$ & 2.32 & 11.00 \\
\hline 40 & 741965 & 1 & 744410 & 12 & 3 & $8,16,16$ & 0.33 & 12.00 \\
\hline 48 & 599373 & 3 & 622678 & 14 & 3 & $16,16,16$ & 3.89 & 4.67 \\
\hline 56 & 514688 & 3 & 571569 & 17 & 3 & $16,16,24$ & 11.05 & 5.67 \\
\hline 64 & 473997 & 2 & 492899 & 27 & 3 & $16,16,32$ & 3.99 & 13.50 \\
\hline
\end{tabular}

\begin{tabular}{|c|c|c|c|c|c|c|c|c|}
\hline \multirow[b]{2}{*}{$\underline{W}$} & \multicolumn{2}{|c|}{ Method in [7] } & \multicolumn{4}{|c|}{ Hierarchical method } & \multirow[b]{2}{*}{$\Delta \mathcal{T}(\%)$} & \multirow[b]{2}{*}{$\frac{\mathcal{E}_{\text {hies }}}{\mathcal{E}_{\text {flat }}}$} \\
\hline & $\mathcal{T}_{\text {flat }}$ & $\mathcal{E}_{\text {flat }}$ & $\mathcal{T}_{\text {hier }}$ & $\mathcal{E}_{\text {hier }}$ & $B_{\text {hier }}$ & Part $_{\text {hier }}$ & & \\
\hline 16 & 41547400 & 9 & 45081900 & 15 & 2 & 5,11 & 1.29 & 1.67 \\
\hline 24 & 28399400 & 13 & 30630900 & 16 & 2 & 8,16 & 7.86 & 1.23 \\
\hline 32 & 21040300 & 18 & 22944500 & 18 & 2 & 8,24 & 9.05 & 1.00 \\
\hline 40 & 19144300 & 19 & 22944500 & 19 & 2 & 8,32 & 19.85 & 1.00 \\
\hline 48 & 15212400 & 19 & 21140200 & 22 & 2 & 8,40 & 39.00 & 1.16 \\
\hline 56 & 14090700 & 29 & 19225700 & 26 & 2 & 8,48 & 36.44 & 0.90 \\
\hline 64 & 14090700 & 34 & 19225700 & 28 & 2 & 8,56 & 36.44 & 0.82 \\
\hline
\end{tabular}

In Table I(c), we present results for p93791. Hierarchical TAM optimization was performed using the heuristic method [19]. A TAM width of 16 was supplied to each megacore prior to system-level TAM optimization. In Table I(d), we present results for a586710. Hierarchical TAM optimization was performed using ILP and enumeration [7]. A TAM width of eight bits $\left(W_{i}=W_{i}^{\star}=8\right.$ for megacore $\left.i\right)$ was supplied to each megacore prior to system-level TAM optimization.

\section{B. Interactive Design Transfer Model}

We next carried out TAM optimization using the design flow in Fig. 4. A set of potential TAM widths and corresponding 
TABLE IV

ILLUSTRATION OF THE DEPENDENCE OF SOC-LEVEL TAM DESIGN ON INTERNAL TAM WIDTHS OF MEGACORES FOR P34392

\begin{tabular}{c|c|c|c|c|c|c|c|c|c}
\hline & $\begin{array}{c}W_{1} \\
W\end{array}$ & $\begin{array}{c}W_{2} \\
\text { (Mega-core 1) }\end{array}$ & $\begin{array}{c}W_{3} \\
\text { (Mega-core 2) }\end{array}$ & $\begin{array}{c}\text { SOC testing } \\
\text { (Mega-core 3) }\end{array}$ & time $\mathcal{T}$ & $B_{\text {hier }}$ & por TAM & & \\
\hline 32 & 8 & 8 & 8 & 815943 & 3 & $5,8,19$ & 8 & 5 & 8 \\
& 12 & 12 & 747814 & 3 & $6,10,16$ & 12 & 6 & 10 \\
& 16 & 16 & 788873 & 2 & 16,16 & 16 & 16 & 16 \\
& 20 & 20 & 780876 & 3 & $6,12,14$ & 14 & 6 & 12 \\
& 24 & 24 & 756204 & 3 & $6,12,14$ & 14 & 6 & 12 \\
\hline 40 & 12 & 12 & 12 & 747814 & 3 & $6,10,24$ & 12 & 6 & 10 \\
& 16 & 16 & 618597 & 3 & $6,16,18$ & 16 & 6 & 16 \\
& 20 & 20 & 667943 & 3 & $5,16,19$ & 16 & 5 & 19 \\
& 24 & 24 & 24 & 643271 & 3 & $5,16,19$ & 19 & 5 & 16 \\
\hline 40 & 8 & 16 & 24 & 815943 & 3 & $4,13,23$ & 8 & 4 & 13 \\
\hline 40 & 12 & 24 & 16 & 747814 & 3 & $6,10,24$ & 12 & 6 & 10 \\
\hline 40 & 24 & 8 & 20 & 658165 & 3 & $5,15,20$ & 15 & 5 & 20 \\
\hline
\end{tabular}

TABLE V

ILLUSTRATION OF THE DEPENDENCE OF SOC-LEVEl TAM DESIGN ON INTERNAL TAM WIDTHS OF MEGACORES FOR P93791

\begin{tabular}{c|c|c|c|c|c}
\hline & & & & SOC TAM \\
$W$ & $\left(W_{1}, W_{2}, W_{3}, W_{4}, W_{5}, W_{6}, W_{7}, W_{8}\right)$ & $\mathcal{T}$ & $B_{\text {hier }}$ & partitions & $\left(W_{1}^{\star}, W_{2}^{\star}, W_{3}^{\star}, W_{4}^{\star}, W_{5}^{\star}, W_{6}^{\star}, W_{7}^{\star}, W_{8}^{\star}\right)$ \\
\hline 32 & $(8,8,8,8,8,8,8,8)$ & 996252 & 6 & $4,4,4,4,7,9$ & $7,8,7,4,4,4,4,4$ \\
& $(12,12,12,12,12,12,12,12)$ & 915300 & 4 & $2,9,9,12$ & $9,12,9,9,9,9,9,12$ \\
& $(16,16,16,16,16,16,16,16)$ & 915082 & 3 & $7,9,16$ & $9,16,7,16,16,9,7,9$ \\
& $(20,20,20,20,20,20,20,20)$ & 977457 & 4 & $4,4,9,15$ & $15,15,4,4,15,4,9,9$ \\
\hline 40 & $(24,24,24,24,24,24,24,24)$ & 923188 & 2 & 9,23 & $23,23,23,9,9,23,23,9$ \\
& $(12,12,12,12,12,12,12,12)$ & 746107 & 5 & $8,8,8,8,8$ & $8,8,8,8,8,8,8,8$ \\
& $(20,20,20,20,20,20,20,20)$ & 745659 & 4 & $8,8,8,16$ & $16,16,8,8,16,16,16,16$ \\
\hline 40 & $(24,24,24,24,24,24,24,24)$ & 750486 & 2 & 16,24 & $16,8,8,16,8,16,16,8$ \\
\hline 40 & $(16,24,12,8,24,8,20,8)$ & 745710 & 5 & $8,8,8,8,8$ & $16,24,16,24,16,24,24,24$ \\
\hline 40 & $(26,23,17,11,5,24,18,12)$ & 847716 & 4 & $10,10,10,10$ & $8,8,8,8,8,8,8,8$ \\
\hline 40 & $(16,27,25,24,22,21,19,17)$ & 747094 & 4 & $8,8,8,16$ & $8,8,8,8,8,8,8,8$ \\
\hline 40 & $(23,17,5,18,6,20,8,21)$ & 789264 & 5 & $5,8,8,9,10$ & $10,10,10,10,5,10,10,10$ \\
\hline 40 & $(28,16,28,16,28,16,28,15)$ & 741514 & 3 & $8,16,16$ & $16,16,8,8,8,8,8,8$ \\
\hline 40 & $(4,11,18,25,7,14,21,28)$ & 956737 & 5 & $5,5,5,5,20$ & $9,10,5,9,5,8,8,9$ \\
\hline
\end{tabular}

testing times for each megacore was calculated prior to systemlevel TAM optimization. The best TAM width for each megacore was identified at the system level and the final SOC testing time was then determined. In Table II, we compare the testing times and CPU times for hierarchical TAM optimization with those for the corresponding flat methods in [7], and [19]. We do not list the testing times and CPU times of the flat methods, since these are already listed in Table I. We also compare the testing times for the interactive design transfer model with the testing times for the noninteractive model.
In Table II(a), we present results for p22810. The hierarchical TAM optimization flow was based on the ILP and enumeration method of [7]. The testing times obtained are very close to those for flat TAM design, thereby demonstrating that multilevel TAM optimization for hierarchical SOCs can indeed be performed with effective results. More importantly, testing times here are significantly lower than those for the noninteractive case [see last column of Table II(a)]. This is a result of the greater flexibility in choosing lower-level TAM widths on the basis of system-level optimization. 
In Table II(b), we present results for p34392. Hierarchical TAM optimization was performed based on the heuristic method of [19]. The testing times are once again lower than those for the noninteractive case.

In Table II(c), we present hierarchical TAM optimization results for p93791 performed using the heuristic method [19]. The increase in testing time over the flat case ranges from $1.67 \%$ to $7.88 \%$, except for $W=32$, for which a small decrease in testing time of $3.42 \%$ over the flat case is observed. We attribute this anomaly to the fact that the method in [19] is heuristic and inefficient TAM width assignments can sometimes be made when the search-space is large. Finally, in Table II(d), we present results for a586710. Hierarchical TAM optimization was performed using ILP and enumeration [7]. Testing times were equal or lower than those obtained for the noninteractive case.

\section{TAM Optimization for Scenario 3}

We next used the design flow of Fig. 7 to carry out TAM optimization for the four hierarchical SOCs. As in the case of noninteractive design transfer (Scenario 2), the system integrator has no flexibility in designing the internal TAM architecture of the megacores. An important difference, however, lies in the fact that the system integrator can carry width adaptation for the megacores by designing their wrappers. Once again, TAM optimization was carried out using the methods presented in [7], [19].

Table III(a) present results for p22810. The hierarchical TAM optimization flow was based on the ILP and enumeration method of [7]. The internal TAM width for each megacore is set to eight bits $\left(W_{i}=8\right.$ for megacore $\left.i\right)$. The external TAM width $W_{i}^{\star}$ for each megacore $i$ is determined by TAM optimization procedure, and the wrapper for the megacore provides eight bits internally from the $W_{i}^{\star}$ bits fed to it at the SOC level. The testing times here are slightly higher than those obtained in [7] and for the interactive Scenario 2 case, but as expected, they are lower than for the noninteractive Scenario 2 case.

In Table III(b), we present results for p34392. The hierarchical TAM optimization flow was based on the heuristic method of [19]. The testing times here are very close to those reported in [19]. The internal TAM width for the megacores here is set to 16 , thereby providing greater flexibility for TAM optimization at the SOC level. The added flexibility is especially important for this SOC because it has only three megacores and only one top-level nonhierarchical core. Hence, the megacores influence the testing time much more for this SOC than in p22810, which has 20 top-level nonhierarchical cores and only two megacores. Table IV illustrates the dependence of the SOC TAM architecture and the testing time on the number of internal TAM wires in the megacores $\left(W_{1}, W_{2}, W_{3}\right)$. The impact of the top-level TAM width on the external TAM widths of the megacores $\left(W_{1}^{\star}, W_{2}^{\star}, W_{3}^{\star}\right)$ is also shown. Note that the lower bound of 618597 cycles is reached with $W=40$ for hierarchical TAM optimization using Scenario 3. This is in contrast to the interactive case in Scenario 2, for which the lower bound was reached with $W=48$.

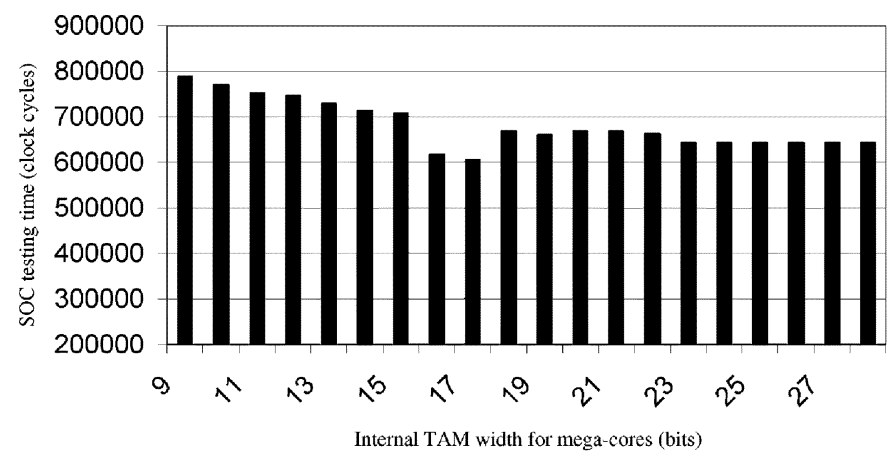

(a)

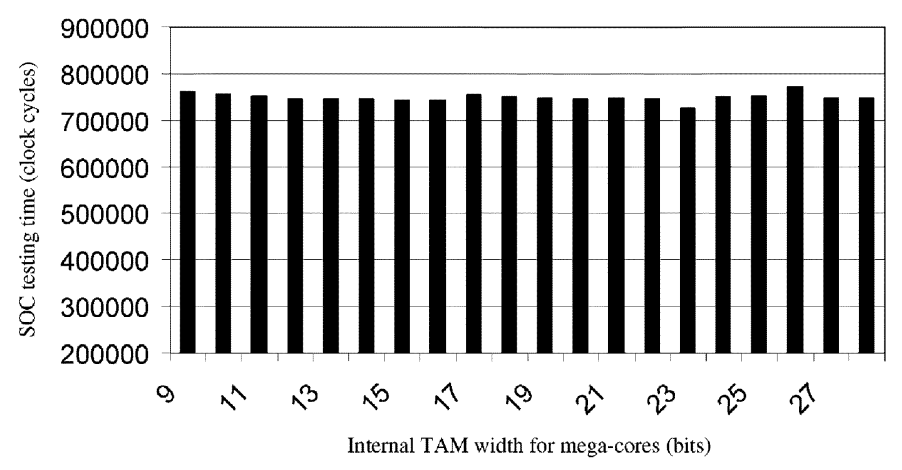

(b)

Fig. 9. Variation of the SOC testing with the internal TAM width of the megacores with $W=40$ for (a) p34392 and (b) p93791.

TABLE VI

VARIATION OF THE TESTING TIME OF A MEGACORE IN P34392 WITH EXTERNAL TAM WIDTH

\begin{tabular}{c|c|c|c|c}
\hline & & & $\begin{array}{c}\text { Total test time } T \\
\left(T_{\text {int }}+T_{\text {ext }}\right)\end{array}$ & $T_{\text {int }} / T$ \\
\hline$W_{i}^{\star}$ & $T_{\text {int }}$ & $T_{\text {ext }}$ & 3309388 & 0.290 \\
4 & 960496 & 2348892 & 2425030 & 0.354 \\
5 & 858949 & 1566081 & 1665531 & 0.294 \\
6 & 490828 & 1174703 & 1420103 & 0.338 \\
7 & 368121 & 876529 & 1244650 & 0.295 \\
8 & 360186 & 876014 & 1236200 & 0.291 \\
9 & 245414 & 587609 & 833023 & 0.295 \\
10 & 245414 & 584524 & 829938 & 0.296 \\
11 & 245414 & 584524 & 829938 & 0.296 \\
12 & 240124 & 584524 & 824648 & 0.291 \\
13 & 240124 & 584524 & 824648 & 0.291 \\
14 & 240124 & 584009 & 824133 & 0.291 \\
15 & 240124 & 583494 & 823618 & 0.292 \\
16 & 122707 & 315179 & 437886 & 0.280 \\
\hline
\end{tabular}

In Table III(c), we present hierarchical TAM optimization results for p93791 performed using the heuristic method of [19] under Scenario 3, with $W_{i}=16$ for each megacore. The testing times here are very close to that obtained for the flat method, 


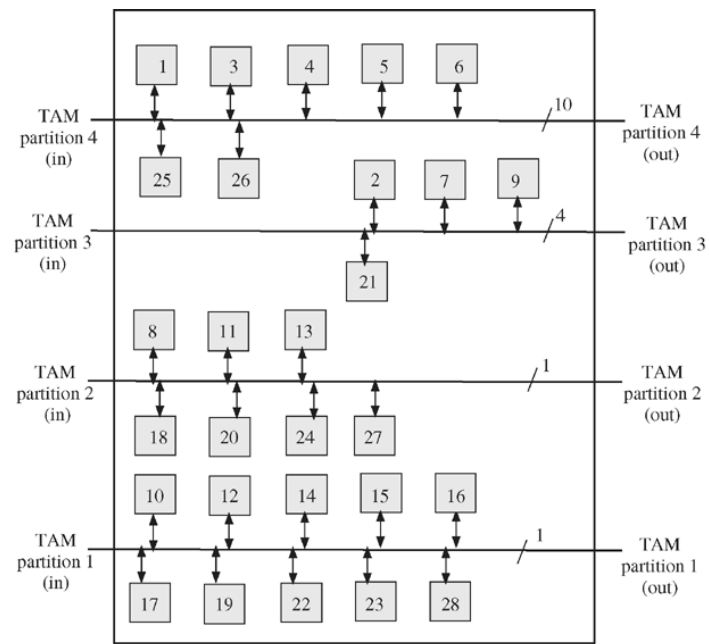

Testing time: 468011 clock cycles

(a)

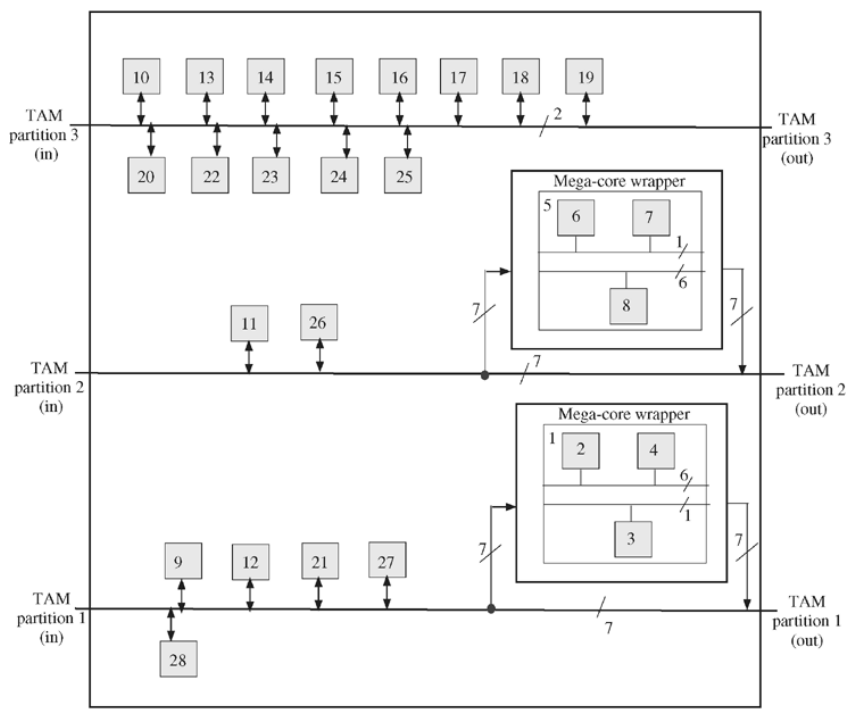

Testing time: 478902 clock cycles

(c)

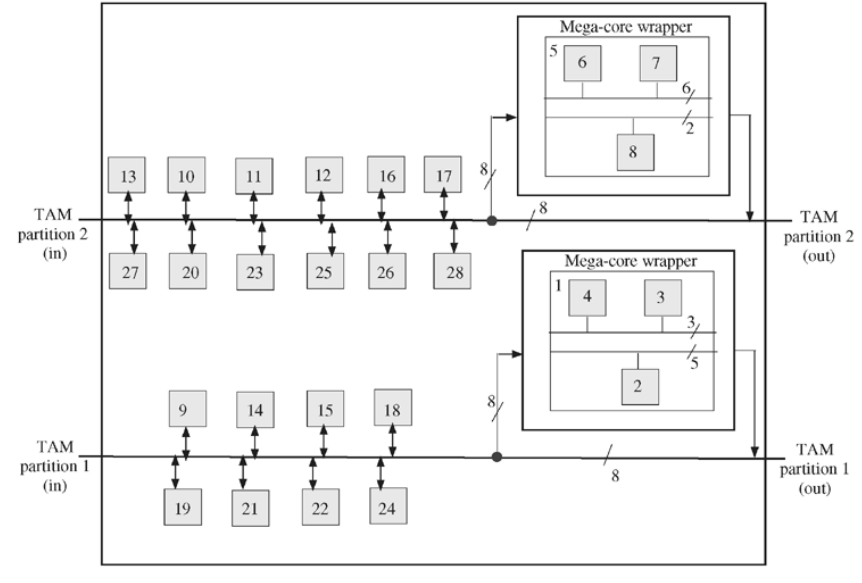

Testing time: 518182 clock cycles

(b)

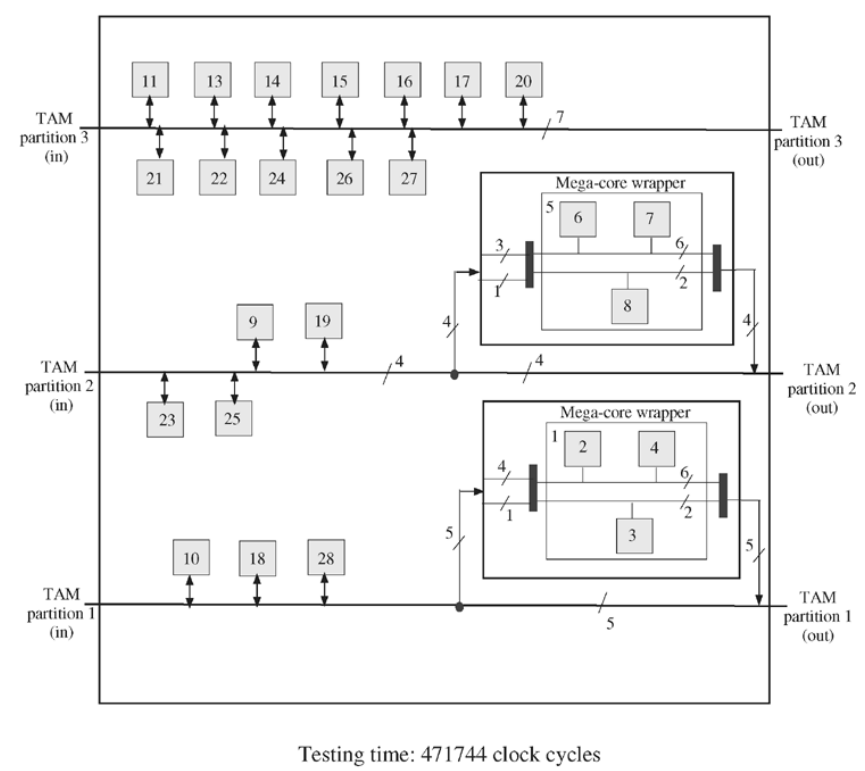

(d)

Fig. 10. Illustration of the TAM architecture for p22810 assuming (a) flat design, (b) noninteractive Scenario 2, (c) interactive Scenario 2, and (d) Scenario 3.

but the computation times, which include the time to obtain the wrapper configurations, tend to be an order of magnitude greater. This can be attributed to the preprocessing time necessary to generate the wrapper configurations for the eight megacores in p93791. In addition to the eight megacores, this SOC also has six top-level nonhierarchical embedded cores. As a result, it offers interesting insights into TAM optimization under Scenario 3. Table V illustrates the dependence of $W$ and the internal TAM widths of the megacores on the SOC testing time and the TAM architecture.

Table III(d) presents results under Scenario 3 for a586710, with $W_{i}=8$. This SOC has one megacore and three top-level, nonhierarchical cores, all of which have large testing times. As a result, the loss of flexibility arising from the predesigned TAM architecture for the megacore leads to a large increase in the testing time compared to the flat case.

Tables IV and V present the impact of $W$ and the internal TAM widths of the megacores on the SOC testing time and the TAM architecture. As illustrated in Fig. 9, for $W=40$, the overall SOC testing time varies significantly for p34392 as the internal TAM widths of the megacores are varied. The variation is much less pronounced for p93791. Similar results were obtained for other representative values of $W$.

We next examine the effect of test data serialization at Wrapper 1 and Wrapper 2 on the testing time of a megacore. Table VI lists the testing times for one of the three megacores of p34392 for various values of the external TAM width $W_{i}^{\star}$, when the internal TAM width is fixed at 16 bits. We separately 
list the testing times for the embedded cores $\left(T_{\mathrm{int}}\right)$ and the logic that is external to the megacores $\left(T_{\text {ext }}\right)$. The results show that both of these testing times decrease at the same rate, keeping the ratio $T_{\text {int }} / T_{\text {ext }}$ almost constant. For this megacore, $T_{\text {ext }}$ is much larger than $T_{\text {int }}$ because, while the megacore has 29 top-level scan chains, the seven embedded cores have no internal scan chains.

Finally, in Fig. 10, we illustrate the TAM architectures obtained for the various scenarios with $W=16$ for $\mathrm{p} 22810$. Using the optimization technique presented in [19]. In Fig. 10(b), we assume an internal TAM width of eight bits for the megacores. An optimal TAM width of seven bits is determined for the megacores in Fig. 10(c). In Fig. 10(d), we assume that each megacore is provided with an internal TAM width of eight bits by the megacore vendor. Appropriate external TAM partitions for the megacores are determined during SOC-level TAM optimization. We note that, in this case, lower testing time is obtained using Scenario 3 compared to interactive Scenario 2. The lack of flexibility due to the rigid TAM architecture for the megacores in Scenario 3 is offset by the added freedom of TAM partitioning at the SOC level.

\section{CONCLUSION}

We have shown how TAM optimization methods proposed for "flattened SOCs" can be used to solve the more realistic problem of designing multilevel TAM architectures for hierarchical SOCs containing megacores. Several test-planning scenarios have been addressed in this paper. These scenarios correspond to the level of interaction between the megacore vendor and the system integrator. Three TAM optimization flows have been proposed for these scenarios, which are directly applicable to real-world design transfer models used by core vendors and SOC integrators. Experimental results for benchmark SOCs indicate that testing times using hierarchical TAMs are often comparable to those achieved using flat methods. As part of ongoing work, we are developing a more general optimization framework for Scenario 3 to merge Wrappers 1 and 2 of the megacores into a unified wrapper, which provides lower testing time for the hierarchical SOC. We are also investigating power constraints and place-and-route issues in hierarchical test planning.

\section{ACKNOWLEDGMENT}

The authors thank E. J. Marinissen and S. K. Goel of Philips Research Laboratories, and A. Sehgal of Duke University for valuable discussions, as well as for comments on a draft version of this manuscript. They also thank the anonymous reviewers for valuable comments; in particular, they thank Reviewer 2 for bringing to our attention references [26] and [27], and Reviewer 3 for suggesting the use of reconfigurable wrappers and multiple core instances.

\section{REFERENCES}

[1] Semiconductor Industry Association. (2003) International Technology Roadmap for Semiconductors (ITRS). [Online]. Available: http://public.itrs.net/Files/2003ITRS/Home2003.htm
[2] V. Chickermane et al., "A building block BIST methodology for SOC designs: A case study," in Proc. Int. Test Conf., 2001, pp. $111-120$

[3] Y. Zorian, E. J. Marinissen, and S. Dey, "Testing embedded-core-based system chips," IEEE Comput., vol. 32, no. 6, pp. 52-60, Jun. 1999.

[4] P. Harrod, "Testing re-usable IP: A case study," in Proc. Int. Test Conf., 1999, pp. 493-498.

[5] E. J. Marinissen et al., "A structured and scalable mechanism for test access to embedded reusable cores," in Proc. Int. Test Conf., 1998, pp. 284-293.

[6] P. Varma and S. Bhatia, "A structured test re-use methodology for corebased system chips," in Proc. Int. Test Conf., 1998, pp. 294-302.

[7] V. Iyengar, K. Chakrabarty, and E. J. Marinissen, "Test wrapper and test access mechanism co-optimization for system-on-chip," J. Electron. Testing: Theory Applicat., vol. 18, pp. 213-230, 2002.

[8] Y. Huang et al., "On concurrent test of core-based SOC design," J. Electron. Testing: Theory Applicat., vol. 18, pp. 401-414, 2002.

[9] S. K. Goel and E. J. Marinissen, "Effective and efficient test architecture design for SOCs," in Proc. Int. Test Conf., 2002, pp. 529-538.

[10] E. Larsson and Z. Peng, "Test scheduling and scan-chain division under power constraint," in Proc. Asian Test Symp., 2001, pp. 259-264.

[11] _ "An integrated framework for the design and optimization of SOC test solutions," J. Electron. Testing: Theory Applicat., vol. 18, pp. 385-400, 2002.

[12] Y. Huang, S. M. Reddy, W. T. Cheng, P. Reuter, N. Mukherjee, C.-C. Tsai, O. Samman, and Y. Zaidan, "Optimal core wrapper width selection and SOC test scheduling based on 3-D bin packing algorithm," in Proc. Int. Test Conf., 2002, pp. 74-82.

[13] W. Zou, S. M. Reddy, I. Pomeramz, and Y. Huang, "SOC test scheduling using simulated annealing," in Proc. VLSI Test Symp., 2002, pp. $325-330$.

[14] S. Koranne, "On test scheduling for core-based SOCs," in Proc. Int. Conf. VLSI Design, 2002, pp. 505-510.

[15] I. Parulkar, T. Ziaja, R. Pendurkar, A. D’Souza, and A. Majumdar, “A scalable, low cost design-for-test architecture for UltraSPARC chip multi-processors," in Proc. Int. Test Conf., 2002, pp. 726-735.

[16] T. J. Chakraborty, S. Bhawmik, and C.-H. Chiang, "Test access methodology for system-on-chip testing," in Proc. Int. TECS Workshop, 2000, pp. 1.1-1-1.1-7.

[17] M. Benabdenbi, W. Maroufi, and M. Marzouki, "CAS-BUS: A test access mechanism and a toolbox environment for core-based system chip testing," J. Electron. Testing: Theory Applicat., vol. 18, pp. 455-473, 2002.

[18] A. Benso et al., "HD2BIST: A hierarchical framework for BIST scheduling, data patterns delivering and diagnosis in SOCs," in Proc. Int. Test Conf., 2000, pp. 892-901.

[19] V. Iyengar, K. Chakrabarty, and E. J. Marinissen, "Efficient wrapper/TAM co-optimization for large SOCs," in Proc. Design Automation Test Eur. Conf., 2002, pp. 491-498.

[20] E. J. Marinissen, S. K. Goel, and M. Lousberg, "Wrapper design for embedded core test," in Proc. Int. Test Conf., 2000, pp. 911-920.

[21] E. J. Marinissen, V. Iyengar, and K. Chakrabarty, "A set of benchmarks for modular testing of SOCs," in Proc. Int. Test Conf., 2002, pp. 519-528.

[22] E. J. Marinissen, R. Kapur, M. Lousberg, T. McLaurin, M. Ricchetti, and Y. Zorian, "On IEEE P1500's standard for embedded core test," $J$. Electron. Testing: Theory Applicat., vol. 18, pp. 365-383, 2002.

[23] T. H. Cormen, C. E. Leiserson, and D. L. Rivest, Introduction to Algorithms. New York: McGraw-Hill, 2001.

[24] V. Iyengar, K. Chakrabarty, and E. J. Marinissen, "On using rectangle packing for SOC wrapper/TAM co-optimization," in Proc. VLSI Test Symp., 2002, pp. 253-258.

[25] S. Koranne, "A novel reconfigurable wrapper for testing of embedded core-based SOC's and its associated scheduling algorithm," J. Electron. Testing: Theory Applicat., vol. 18, pp. 415-434, 2002.

[26] Y. Wu and P. MacDonald, "Testing ASIC's with multiple identical cores," IEEE Trans. Computer-Aided Design Integr. Circuits Syst., vol. 22, no. 3, pp. 327-336, Mar. 2203.

[27] Y. Huang, S. M. Reddy, and W. T. Cheng, "Core-clustering based SOC test scheduling optimization," in Proc. Asian Test Symp., 2002, pp. $405-410$. 


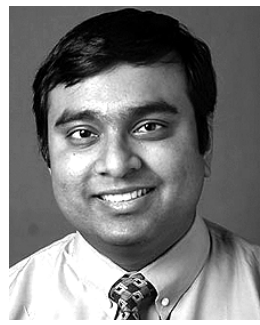

Krishnendu Chakrabarty (S'92-M'96-SM'00) received the B.Tech. degree from the Indian Institute of Technology, Kharagpur, India, in 1990, and the M.S.E. and Ph.D. degrees from the University of Michigan, Ann Arbor, in 1992 and 1995, respectively, all in computer science and engineering.

$\mathrm{He}$ is currently an Associate Professor of Electrical and Computer Engineering at Duke University, Durham, NC. From 2000 to 2002, he was also a Mercator Visiting Professor at the University of Potsdam, Potsdam, Germany. His current research projects include: design and testing of system-on-a-chip integrated circuits; embedded real-time systems; distributed sensor networks; design automation methods for microfluidics-based biochips; microfluidics-based chip cooling. $\mathrm{He}$ is a coauthor of two books: Microelectrofluidic Systems: Modeling and Simulation (Boca Raton, FL: CRC, 2002) and Test Resource Partitioning for System-on-a-Chip (Norwell, MA: Kluwer, 2002), and an editor of SOC (System-on-a-Chip) Testing for Plug and Play Test Automation (Norwell, MA: Kluwer, 2002). He has published over 170 papers in journals and refereed conference proceedings, and holds a U.S. patent in built-in self-test.

Dr. Chakrabarty is a Member of ACM, ACM SIGDA, and Sigma Xi. He is a recipient of the National Science Foundation Early Faculty (CAREER) Award and the Office of Naval Research Young Investigator Award. He received a Best Paper Award at the 2001 Design, Automation, and Test in Europe (DATE) Conference. He is also the recipient of the Humboldt Research Fellowship, awarded by the Alexander von Humboldt Foundation, Germany. $\mathrm{He}$ is an Associate Editor of IEEE TRANSACTIONS ON COMPUTER-AIDED DESIGN OF INTEGRATED CIRCUITS AND SYSTEMS, an Editor of the Journal of Electronic Testing: Theory and Applications (JETTA), and a member of the editorial boards of the ACM Journal of Emerging Technologies in Computing Systems, Sensor Letters and the Journal of Embedded Computing. He has also served as an Associate Editor of the IEEE TRANSACTIONS ON CIRCUITS AND Systems-PART II: ANALOG AND Digital Signal Processing. He serves as Vice Chair of Technical Activities of the IEEE's Test Technology Technical Council and is a Member of the program committees of several IEEE/ACM conferences and workshops.

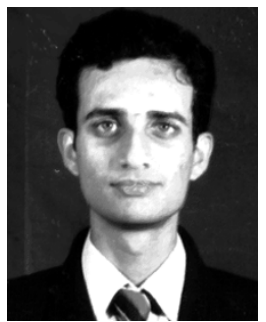

Vikram Iyengar received the B.E. degree in electrical engineering from the Birla Institute of Technology, Mesra, India, in 1996, the M.S. degree in computer engineering from Boston University, Boston, MA, in 1998, and the Ph.D. degree in computer engineering from Duke University, Durham, $\mathrm{NC}$, in 2002.

$\mathrm{He}$ is currently an Advisory Engineer with the ASIC Test Methodology Group, IBM Microelectronics, Essex Junction, VT. He is the co-author of Test Resource Partitioning for System-on-a-Chip (Norwell, MA: Kluwer, 2002). He has published over 30 journal articles and refereed conference papers in the area of VLSI design and test. His research interests lie in system-on-chip design and test.

Dr. Iyengar is a Member of ACM Sigda and Sigma Xi. He was a recipient of the Boston University Electrical and Computer Engineering Department Chair Fellowship Award in 1996, an IBM Graduate Fellowship Award in 2001, and the EDAA Outstanding Dissertation Award in 2004.

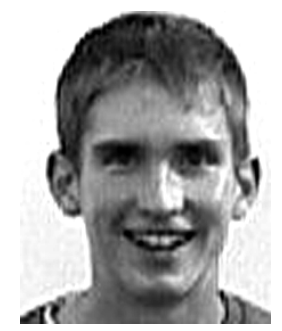

Mark D. Krasniewski received the B.S.E. degree in electrical engineering from Duke University, Durham, NC, in 2003. He is currently pursuing the M.S. degree in computer engineering at Purdue University, West Lafayette, IN.

His interests include very large scale integration design and test and fault tolerance in sensor networks. 